\title{
Charge separation at nanostructured molecular donor-acceptor interfaces
}

Andreas Opitz, Rupak Banerjee, Stefan Grob, Mark Gruber, Alexander Hinderhofer, Ulrich Hörmann, Julia Kraus, Theresa Linderl, Christopher Lorch, Andreas Steindamm, Anna-Katharina Topczak, Andreas Wilke, Norbert Koch, Jens Pflaum, Frank Schreiber, and Wolfgang Brütting

\begin{abstract}
Planar and bulk heterojunctions of organic donor and acceptor molecules are used for understanding elementary processes in photovoltaic cells. Electronic structure, interface and film morphology, excitonic behaviour, device characteristics and correlations between those properties are reviewed here using a wide range of material combinations.
\end{abstract}

\subsection{Introduction to molecular donor-acceptor solar cells}

Organic photovoltaic cells (OPVCs) have recently surmounted the psychologically important barrier of $10 \%$ efficiency for the conversion of incoming solar to electrical power and the current record value for the power conversion efficiency of molecular OPVCs has reached more than $11 \%[1,2]$. The rapid progress over recent years poses the question, where the fundamental efficiency limits of these materials are. For inorganic photovoltaics, this question was answered more than 50 years ago by the well-known Shockley-Queisser (SQ) limit [3]. Based on very few fundamental assumptions, the SQ theory is able to predict an upper limit for the power conversion efficiency of single junction cells with the energy gap $E_{\mathrm{G}}$ of the active semiconductor material as the only input parameter. Owing to their excitonic nature, however, an important modification of this concept is required for OPVCs, where a donor-acceptor (DA) heterojunction is used to enable charge separation. This is related to the existence of a charge transfer (CT) state (see Fig. 4.1) as an intermediate in the dissociation process from the photo-excited (excitonic) state on one molecular species towards free charge separated states on both molecular species $[4-7]$.

The overall charge generation process can be split up into four steps [9] as illustrated in Fig. 4.1: 

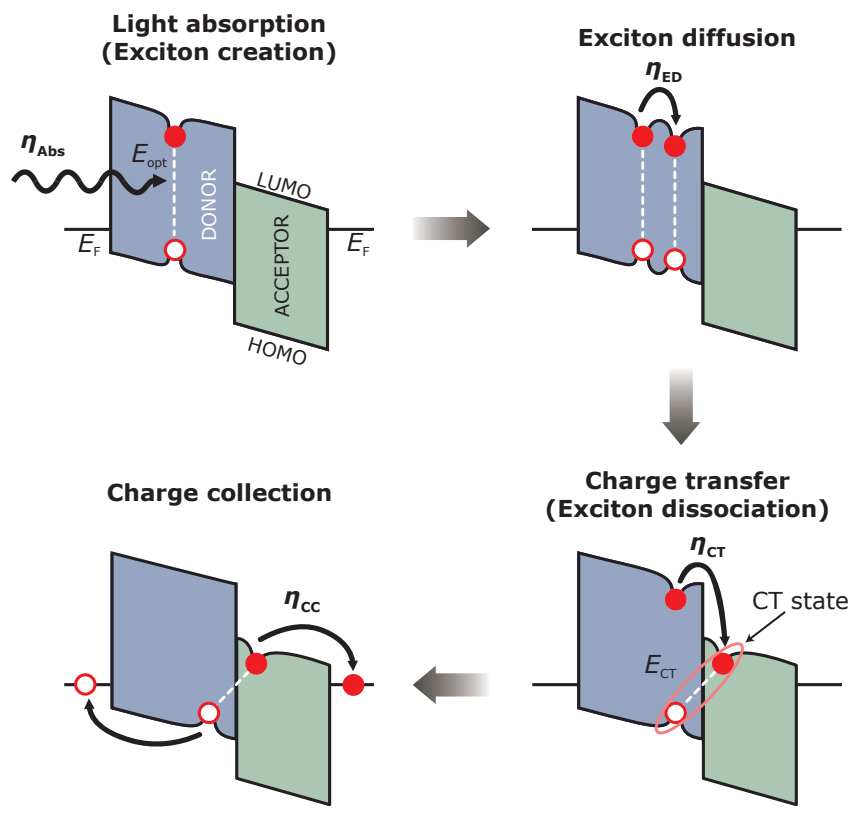

Fig. 4.1. Basic processes in organic solar cells visualized in a schematic energy diagram of a DA cell under short-circuit conditions. $E_{\mathrm{F}}$ are the Fermi energies of the cathode and anode contacts, respectively. LUMO is the lowest unoccupied molecular orbital, and HOMO is the highest occupied molecular orbital of the organic film. Filled circles represent electrons and open circles holes. A line between electron and hole symbolizes an exciton with a dip in the energy levels depicting the lowering of energy by Coulomb interaction between electron and hole. The figure is adapted from $[8]$.

1. Absorption of light with photon energy above the optical gap $E_{\text {opt }}$ and generation of excitons (ideally in both materials, but here shown for simplicity only in the donor).

2. Exciton diffusion from the bulk of the film to the DA interface, where the exciton diffusion length determines the fraction of the layer that is actually relevant.

3. Exciton dissociation and formation of a charge transfer state at the interface, which is still coulombically bound.

4. Charge separation and carrier collection at the electrodes to produce electrical power in the external circuit.

Accordingly, the internal quantum efficiency is given by [9]:

$$
\eta_{\mathrm{int}}=\eta_{\mathrm{Abs}} \cdot \eta_{\mathrm{ED}} \cdot \eta_{\mathrm{CT}} \cdot \eta_{\mathrm{CC}}
$$



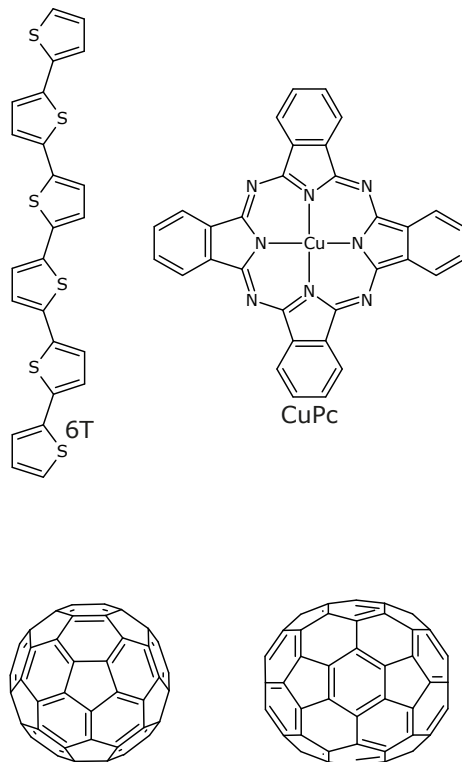

$\mathrm{c} 60$

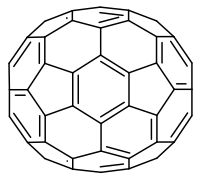

$\mathrm{C} 70$
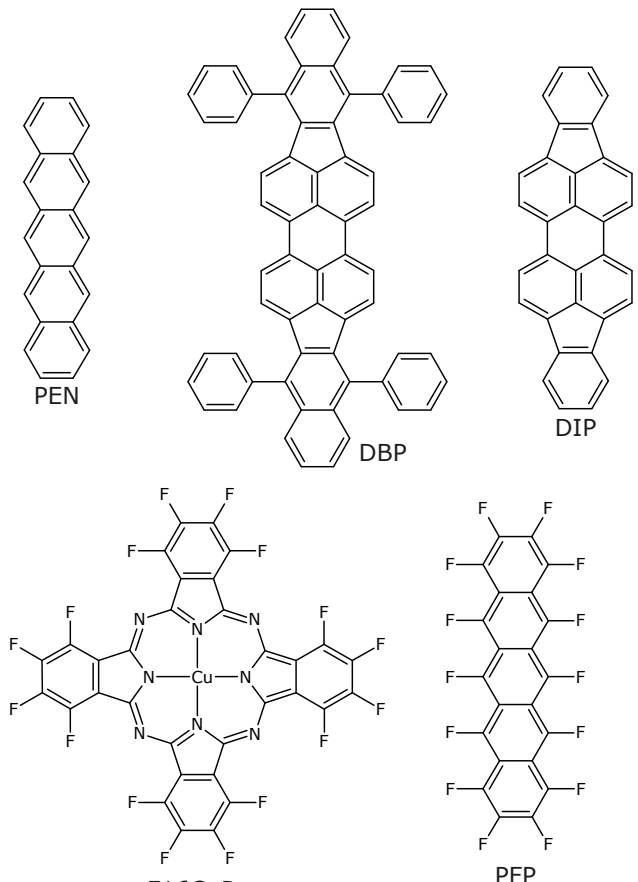

F16CuPc

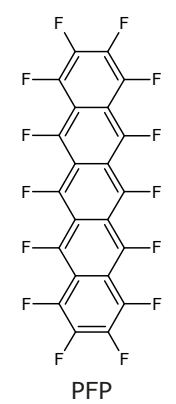

PFP

Fig. 4.2. Chemical structures of molecular materials (6T: $\alpha$-sexithiophene; CuPc: copper phthalocyanine; PEN: pentacene; DBP: tetraphenyldibenzoperiflanthene, DIP: diindenoperylene; C60 and C70: Buckminster fullerenes; F16CuPc: perfluorinated $\mathrm{CuPc}$; PFP: perfluorinated $\mathrm{PEN})$.

which is the product of the absorption efficiency $\eta_{\mathrm{Abs}}$, the exciton diffusion efficiency $\eta_{\mathrm{ED}}$, the charge transfer efficiency $\eta_{\mathrm{CT}}$ and the charge collection efficiency $\eta_{\mathrm{CC}}$.

If reflection losses for coupling light into the cell are taken into account, one obtains the external quantum efficiency (EQE) which is basically the number of collected electrons with respect to the number of incident photons

$$
\eta_{\mathrm{ext}}=(1-R) \cdot \eta_{\mathrm{int}}
$$

with $R$ being the reflectivity of the device. $\eta_{\text {ext }}$ is experimentally determined by the measured current density at short-circuit conditions divided by the incident light intensity at a given wavelength and is also termed incidentphoton-to-current efficiency (IPCE).

The overall power conversion efficiency of a solar cell is given by:

$$
\eta_{\mathrm{P}}=\frac{J_{\mathrm{SC}} \cdot V_{\mathrm{OC}} \cdot \mathrm{FF}}{P_{\mathrm{in}}},
$$




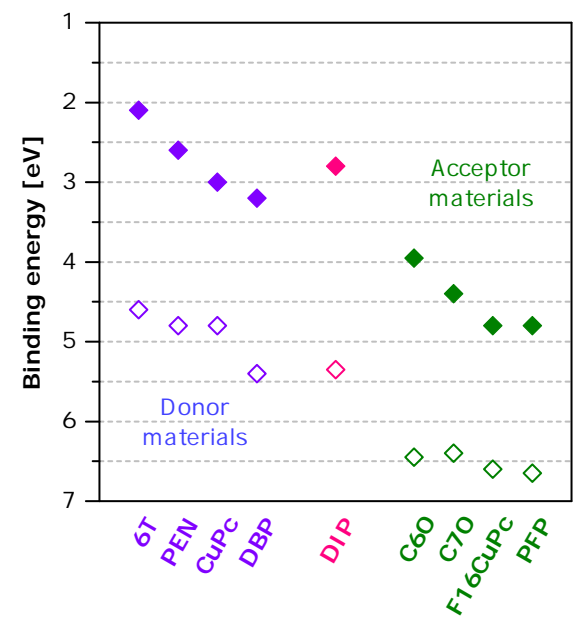

Fig. 4.3. Energy levels of molecular materials in the solid state (closed symbols: electron affinity, open symbols: ionization potential). The IPs were determined by ultraviolet photoelectron spectroscopy (UPS) $[10$ 15]. The EAs are taken from inverse photoelectron spectroscopy [16-19] or from adding the exciton binding energy [20] to the optical gap.

where $J_{\mathrm{SC}}$ is the short-circuit current density, $V_{\mathrm{OC}}$ is the open circuit voltage, $F F$ is the fill factor, and $P_{\text {in }}$ is the incident optical power density, preferably measured under AM1.5 sunlight conditions.

In order to realize different nanomorphologies and investigate their impact on OPVCs, we have chosen a series of molecular donor and acceptor materials from which we prepared of different DA heterojunctions. The chemical structures of $\alpha$-sexithiophene (6T), copper phthalocyanine ( $\mathrm{CuPc}$ ), pentacene (PEN), tetraphenyldibenzoperiflanthene (DBP), diindenoperylene (DIP), Buckminster fullerenes $\mathrm{C} 60$ and $\mathrm{C} 70$, perfluorinated $\mathrm{CuPc}(\mathrm{F} 16 \mathrm{CuPc})$ and perfluorinated PEN (PFP), which were all deposited as thin films by vacuum sublimation, are shown in Fig. 4.2. For all of these materials the energy levels corresponding to the highest occupied molecular orbital (HOMO) and the lowest unoccupied molecular orbital (LUMO) are given as solid-state ionization potential (IP) and electron affinity (EA) in Fig. 4.3. A wide range of energy levels is covered. So long as HOMO and LUMO levels of the acceptor are lying lower than the respective levels of the donor (staggered or type-II heterojunction [21]) and the energy offset between the HOMO and the LUMO levels of the two materials is large enough [22] photovoltaic action appears and the difference between the IP of the donor and the EA of the acceptor - the CT or photovoltaic gap - is an upper limit for $V_{\mathrm{OC}}[22,23]$.

A further material parameter which is important for solar cell performance is the optical absorption spectrum which is obtained from transmission measurements and is shown in Fig. 4.4 for the above mentioned materials. Spectral complements as for $\mathrm{CuPc}$ and $\mathrm{C} 60$ [24] or higher absorption strength of DBP and C70 in comparison to DIP and C60, respectively, [25] are advantageous. However, the interplay between optical absorption, energy levels, morphology, exciton diffusion and transport has to be balanced to improve the overall efficiency of a solar cell. 


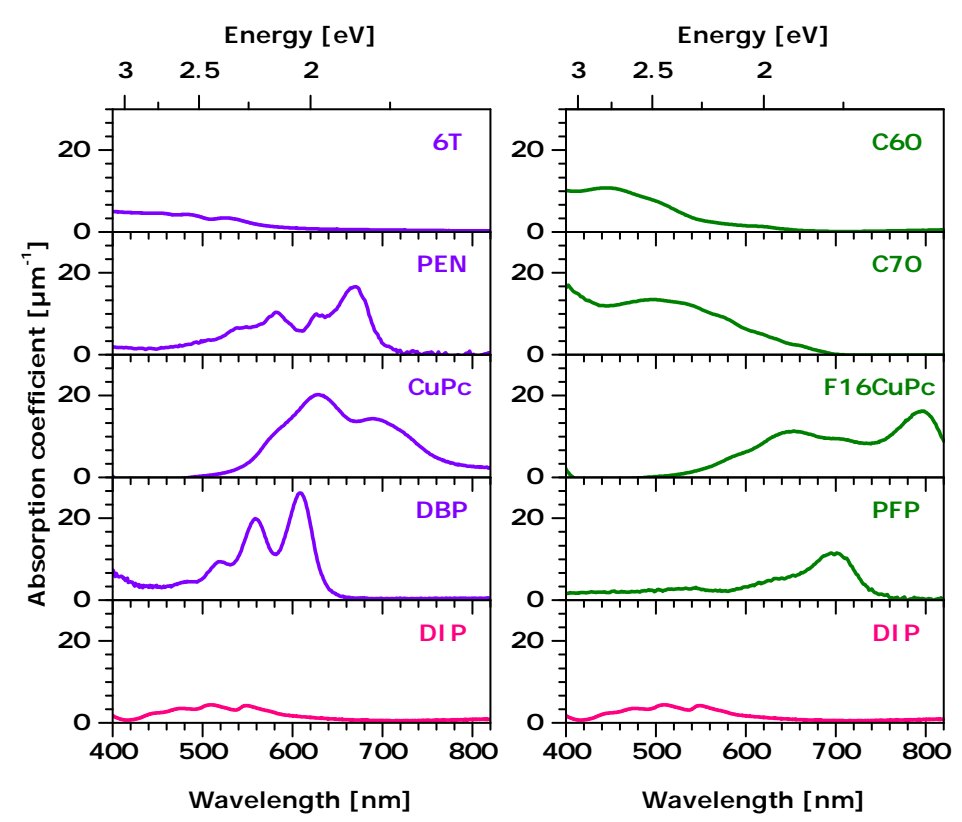

Fig. 4.4. Absorption spectra of the investigated materials (left column: donors; right column: acceptors). The spectra were obtained from transmission measurements on transparent substrates.

Furthermore, the architecture of a solar cell, the arrangement of donor and acceptor molecules in a device, is of importance. Figure 4.5 shows different examples. In all of these architectures an interface to separate the photogenerated excitons is present. The simplest case, as also used by Tang in 1986 [26], is the planar heterojunction (PHJ) where the acceptor layer is deposited on top of the donor film (Fig. 4.5a). A rougher DA interface increases the area where charge separation can take place (Fig. 4.5b). The ideal case is an interdigitated interface with a separation of the columns in the range of the exciton diffusion length (Fig. 4.5c). Often this occurs in a self-organised manner in crystalline materials due to the presence of an intermediate scenario between layer and island growth. The interface area can be increased on purpose also by glancing angle deposition as shown for PEN and DIP [27,28]. Strong phase separation due to structural incompatibility of the two partners, like in the case of PEN or $6 \mathrm{~T}$ co-deposited with $\mathrm{C} 60[8,29]$ can also lead to a morphology close to an interdigitated interface. However, the formation of leakage pathways between the electrodes by one of the materials or very high islands $[8,29]$ is unfavourable for solar cell performance. To cope with low exciton diffusion length the DA interface can be increased by mixing the materials in bulk heterojunctions [30] as illustrated for a molecular 


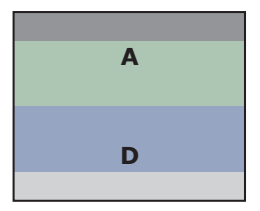

(a)

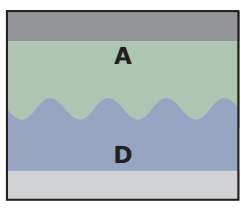

(b)

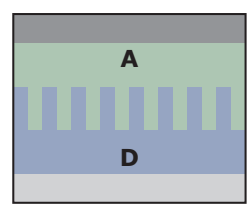

(c)

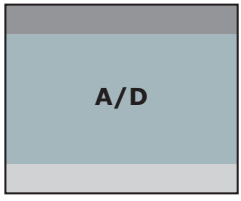

(d)

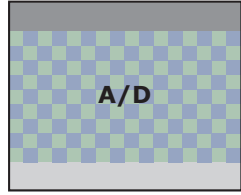

(e)

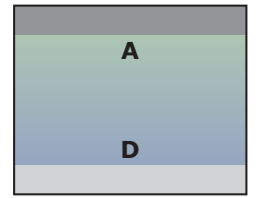

(f)

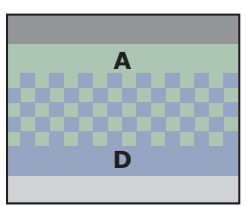

(g)

Fig. 4.5. Different architectures for solar cells: (a) planar DA heterojunction, (b) DA heterojunction with rough interface, (c) DA layers with interdigitated interface, (d) bulk heterojunction (molecular mixture), (e) phase separated bulk heterojunction, (f) gradient heterojunction, (g) planar heterojunction with mixed interface. The figure is adapted from [8].

and a phase-separated mixture in Fig. 4.5d and e, respectively [31]. Molecular mixtures are possible for co-deposition of compounds with similar shapes like CuPc:F16CuPc [32], PEN:PFP [33] or DIP:PFP [34]. Phase separation takes place for co-deposition of molecules with different shapes like $\mathrm{CuPc}$ :C60 [35] or DIP:C60 [12,36]. The advantages of both basic structures, PHJ and BHJ, can be combined by surrounding the mixed film by neat $\mathrm{D}$ and $\mathrm{A}$ layers as shown in Fig. 4.5f for a graded heterojunction and in Fig. 4.5g or a planar-mixed heterojunction (PM-HJ). Thereby the neat layers provide carrier selective contacts and act to some extend also as exciton blocking layer to eliminate exciton quenching at the metal electrodes [25].

To maximise the overall efficiency it is not enough to improve only one of the four processes. Besides balancing the exciton diffusion length with the optical absorption length for the optimal film thickness of absorbing materials, the morphology of the films and the respective alignment of the energy levels are influencing the cell performance as well. In this article we start with an overview on the different aspects of photocurrent generation (electronic structure, interface morphology and phase separation, exciton diffusion and dissociation, charge transport and device performance) to end up with a more conclusive picture including several of these aspects. DIP/C60 will serve as model system throughout this review, but other DA combinations will be touched as well. 


\subsection{Electronic structure}

Ultraviolet and inverse photoelectron spectroscopy (UPS, IPES) were used to determine the electronic structure of interfaces in our OPVCs. These techniques all to assess the distribution of occupied or unoccupied states of the samples [16,38-42]. Typically, a step-wise deposition enables the investigation of the underlying film, the interface as it is being formed, and the on top film as in PHJ. Over the last years also challenges of this procedure were highlighted $[14,43]$. Due to the different electrostatic potentials of the individual layers of the films and the in-plane electrostatics of the surface the properties of the interface buried under a top-layer varies from that observed in the experiment when the interface is incrementally built up. More complexity occurs if the top-deposited film form islands instead of closed layers, for the same reasons. The combination of electrostatic simulations [43] and measurements of energy levels leads to an improved and reliable understanding of the energy level alignment at heterojunctions [44,45]. Furthermore, the detrimental effects of the measurement itself have to be considered. The bombardment of organic materials either with UV photons or low energy electrons can in-
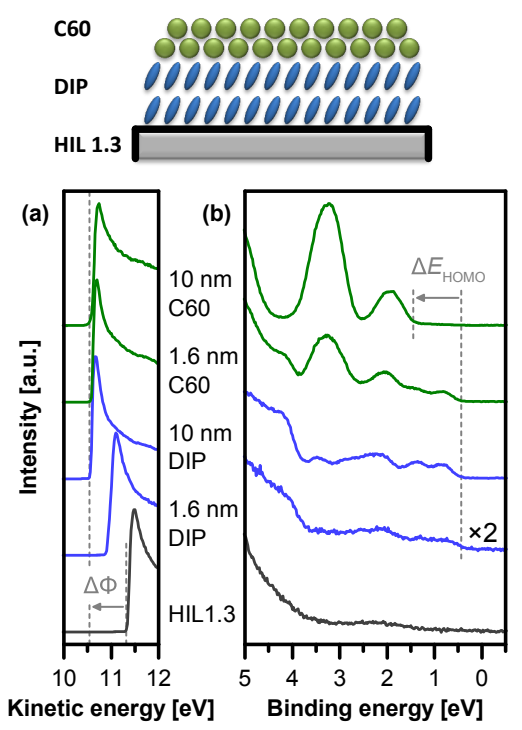

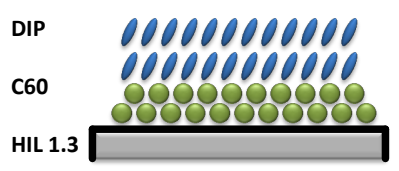

(c)

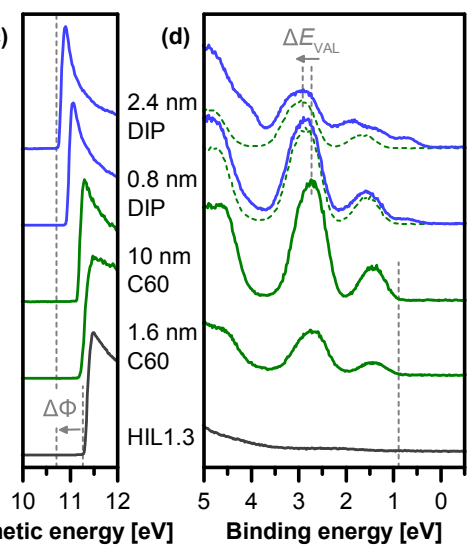

Fig. 4.6. Summary of the secondary electron cut-off and the valence region UPS measurements for the two step-wise deposited layer sequences C60 on DIP (a and b) and DIP on C60 (c and d) with high work function HIL1.3 as underlying contact layer. Vacuum level shifts are marked as $\Delta \Phi$, shift of the valence region as $\Delta E_{\mathrm{VAL}}$, and HOMO level offset as $\Delta E_{\mathrm{HOMO}}$. The dashed lines in part (d) are representing the $\mathrm{C} 60$ content of the spectra. The figure is adapted from $[12,37]$. 


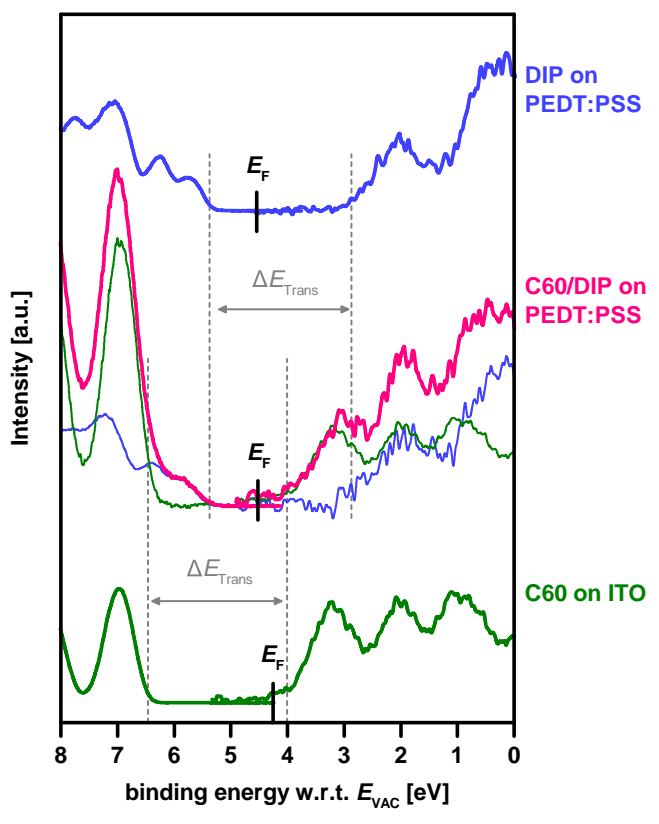

Fig. 4.7. Combined UPS and IPES analysis of pristine layers of DIP and C60 together with the analysis of the PHJ containing a thin C60 layer on top of a DIP film. The spectra of the PHJ can be well described as linear superposition of the pristine film spectra. The transport gaps $\Delta E_{\text {Trans }}$ which are invariant at the interface are for DIP $2.55 \mathrm{eV}$ and for $\mathrm{C} 602.50 \mathrm{eV}$ determined including broadening correction. $E_{\mathrm{F}}$ is the Fermi level for each measurement. The figure is adapted from [17].

duce damage of the molecules and thus irreversible changes of the energy level alignment [14].

In this section the focus lies on the interface between DIP and C60 in both layer sequences. Clevios ${ }^{\mathrm{TM}}$ P AI4083 (designated as PEDT:PSS) and Clevios ${ }^{\text {TM }}$ HIL1.3 (designated as HIL1.3) both purchased from Heraeus Clevios GmbH (Leverkusen, Germany) are used as hole injection layers (HIL) and contact materials.

Figure $4.6 \mathrm{a}$ and $\mathrm{b}$ shows the secondary electron cut-off (SECO) and the valence region UPS spectra for the layer sequence C60 on DIP on top of HIL1.3 $[12,46]$. The shift of the SECO position $\Delta \Phi$ indicates a change of the vacuum level when DIP is deposited on this high work function HIL. In contrast, when PEDT:PSS is used instead of HIL1.3 no change in work function $\Phi$ is observed (not shown) even if the work function of the thick films are the same. The Fermi level is pinned for both HILs inside the DIP only $0.4 \mathrm{eV}$ above the HOMO onset, which represents the hole injection barrier (HIB) magnitude for PEDT:PSS as HIL. The high $\Phi$ HIL1.3 additionally generates a doped contact layer with a reduced HIB of about $0.3 \mathrm{eV}$. The SECO position and with it the work function stays constant after depositing C60 on top of DIP. Nonetheless, the offset between the HOMO onsets of the DIP and the C60 layers $\Delta E_{\text {HOMO }}$ is equal for both of the used HILs.

To analyse also the unoccupied states, combined IPES and UPS measurements were performed on pristine DIP and C60 layers as well as at the 

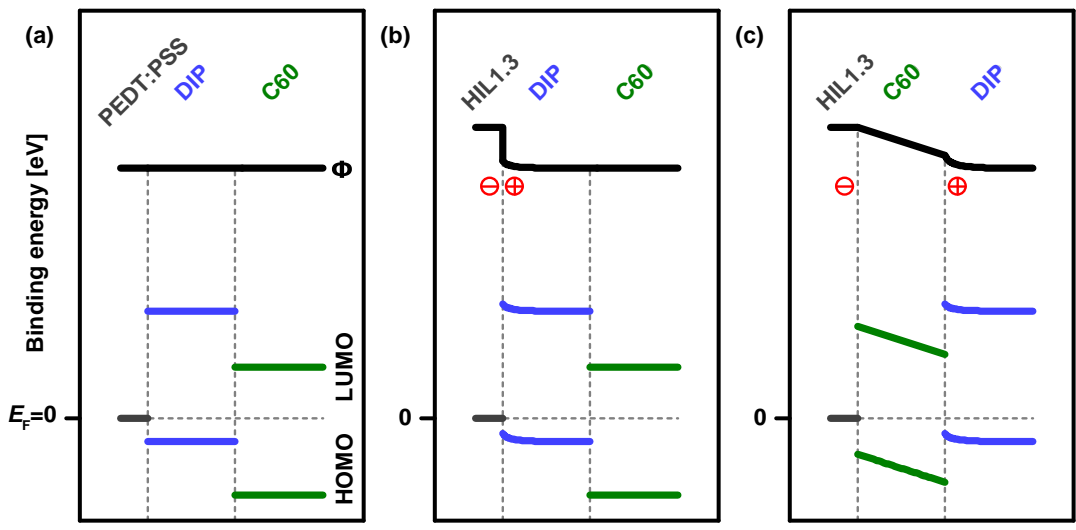

Fig. 4.8. Schematic energy level alignment for three different cases of DIP/C60 planar heterojunctions. (a) Vacuum level alignment at the contact and the organic/organic interface with layer sequence PEDT:PSS/DIP/C60 [12, 14, 46]. (b) Contact doping of the DIP layer with layer sequence HIL1.3/DIP/C60 [12, 14, 46] and vacuum level alignment at the organic/organic interface. (c) Long range charge transfer across the C60 layer with layer sequence HIL1.3/C60/DIP [37].

interface of a thin C60 film deposited on top of a DIP layer [17]. The results are displayed in Fig. 4.7. Deconvolution of the interface spectra is done as a linear superposition of the pristine layer spectra, corrected for a shift of the Fermi level. This shows that the transport gaps of the materials DIP and C60 are $2.55 \mathrm{eV}$ and $2.50 \mathrm{eV}$, respectively, and that they have the same values in pristine films and at the interface between the two materials.

The observed energy level alignment of this layer sequence is summarized in Fig. 4.8a and b $[12,46]$. The reduced HIB when using HIL1.3 results, due to the contact doping, in an improved fill factor of solar cells. S-shape behaviour is present in the solar cells characteristics if the work function is too low and contact doping is absent $[12,46]$. In a detailed study it was shown that the S-shape behaviour can be linked to the work function also when the substrate is heated during the deposition of the DIP layer, essentially due to a lowered water content of the HIL [46]. The injection barrier is relevant at voltages around open circuit conditions because injection and photo-currents (of opposite direction) are balanced at this point. The offset between the HOMO of the donor and the LUMO of the acceptor, the photovoltaic gap, is an upper limit for the open circuit voltage [22,23], which will be discussed further in the summary section 4.6. The vacuum level at the interface is constant in the here presented case, but it can vary by hundreds of meV in general [14]. Any difference changes the photovoltaic gap and therefore the open circuit voltage. The correct value has to be considered for analysing the losses in open circuit voltage in comparison to the photovoltaic gap. Up to now this energy level 


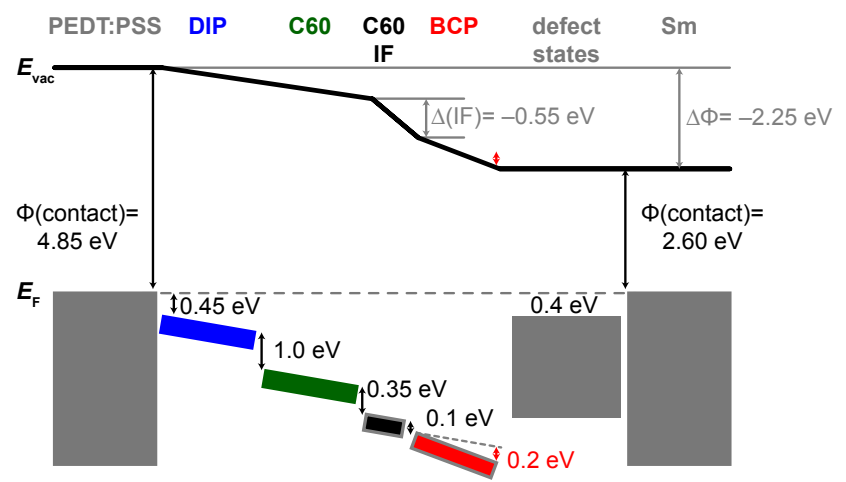

Fig. 4.9. Schematic energy level diagram of the electronic structure across a PEDT:PSS/DIP/C60/BCP/Sm heterojunction photovoltaic cell. The figure is adapted from [14].

alignment cannot be reliably predicted by calculations alone and thus has to be measured.

The UPS measurements for the SECO and valence regions of the layer sequence DIP on C60 on top of HIL1.3 are displayed in Fig. 4.6c and d [37]. The SECO and the HOMO onset positions stay constant during the deposition of C60 on HIL1.3. Upon addition of DIP on top the SECO shifts, indicating a lower work function of the sample. Additionally, the features of the valence region attributed to the fullerene move to higher binding energy. The onset of the HOMO level of DIP is very close to the Fermi level. The Fermi level within the DIP layer is pinned again close to the HOMO level. Like in the case of DIP in direct contact with HIL1.3, the DIP layer is contact-doped, which results from the pinning behaviour. Due to the absence of pinning for the C60 layer itself no charges are induced, giving an intrinsic interlayer. Therefore, the transfer of charges into the DIP layer has to originate from the underlying HIL1.3 (see Fig. 4.8c). This long-range charge transfer happens when the layer in-between the contact and the doped layer is vacuum level aligned with the substrate, which was observed for organic or inorganic interlayers [44, 47]. It has to be noted that a charge transfer between the two organic layers would give similar shifts in the valence region of the underlying material to higher binding energies, which, however, was ruled out for the present case.

Generally, it can be stated that most organic heterojunctions of relevance for OPVCs exhibit no charge transfer in the ground state and thus they feature a constant electrostatic potential across the interface. However, the contact to an electrode may induce Fermi level pinning of one of the organic materials, and depending on layer sequence, may result in long-range charge transfer and potential shifts. This was demonstrated for a high work function electrode (HILs) here above, but this also holds for low work function electrodes, where pinning at the LUMO of one of the heterojunction materials may then occur. 
As an example, the full electronic structure of an OPVC made of a C60/DIP heterojunction sandwiched between PEDT:PSS and Samarium electrodes and including an exciton blocking layer of bathocuproine (BCP) is displayed in Fig. 4.9 [14]. Blocking layers made of BCP or bathophenanthroline (BPhen) were reported to reduce exciton quenching at the metal top contact and they simultaneously serve to protect the C60 layer from metal in-diffusion and damage during evaporation of the top-contact [46, 48,49]. Energy level shifts at the $\mathrm{C} 60 / \mathrm{BCP}$ interface and inside the $\mathrm{BCP}$ layer are due to a preferential orientation of BCP dipoles. The electrostatic field drop across DIP/C60 is therefore reduced compared to the full effective work function difference of the contact materials. Even if the photovoltaic gap at the active interface is unaffected by the electrostatic potential landscape, changes in the electric field distribution throughout the devices is influencing the charge collection efficiency [50].

\subsection{Interface morphology and mixing behaviour}

The morphology and the crystal structure of the active layer is crucially important for the efficiency of organic photovoltaic cells. The orientation of the molecules in the film determines the coupling strength of the transition dipole to the incoming light and hence strongly affects the absorption of the active layer $[51,52]$. The crystal structure and the defect density in the organic films strongly influence the exciton diffusion length and also the charge transport properties of the films, which are vital for the efficiency of the photovoltaic cell. In addition, the interface morphology, especially in PHJ cells, and the degree of phase separation in BHJ cells play a significant role for the efficiency since ideally all of the created excitons should reach the DA interface to be separated.

There are various methods to study the interface and structure of organic thin films. One of the most prominent and widely used technique is atomic force microscopy (AFM). However, AFM is limited to the top surface and it is impossible to obtain information on the structure further below in the film, close to the substrate. Furthermore, it is difficult to distinguish between different materials, i.e. donor and acceptor, due to lack of contrast and hence to determine the phase separation if any. Another suitable technique to investigate thin organic films is X-ray scattering. Different experimental geometries allow the investigation of the out-of-plane structure via X-ray reflectivity (XRR), including the extraction of the electron density profile along the surface normal, the estimation of the surface roughness and the out-of-plane crystallinity of the film. Grazing incidence X-ray diffraction (GIXD) can be used to reveal the in-plane crystal structure of the film and grazing incidence small angle X-ray scattering (GISAXS) allows to determine the morphological features like the surface island distance and their shape $[53,54]$. One of the benefits of $\mathrm{X}$-ray scattering is that it is possible to employ it directly in situ during the 


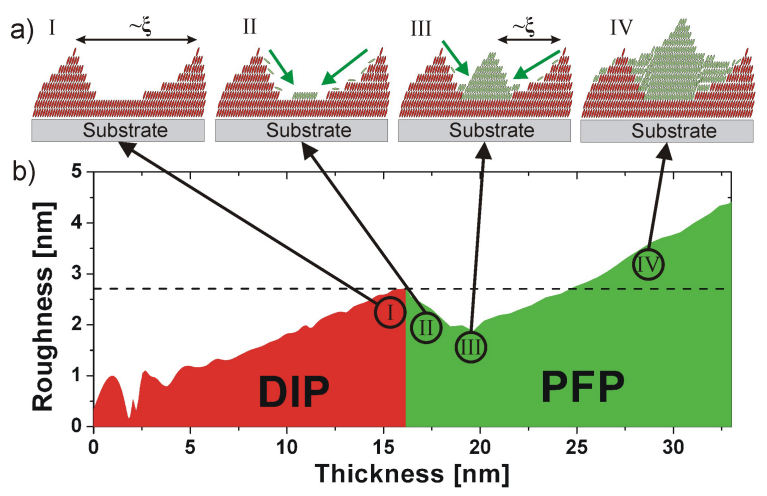

Fig. 4.10. (a) Growth of PFP islands in the voids between the DIP islands leading to a filling of the latter. (b) Roughness evolution of a DIP-PFP heterostructure. Reprinted with permission [55].

preparation of the films and follow the film growth in a non-invasive way in real-time, giving a more detailed understanding of the film growth.

In general, there are several interfaces in organic photovoltaic cells, which are all important for the efficiency. We focus on the DA, so the organicorganic, interface. For details of the interfaces between the active layer and the (metal) electrodes, which is in itself a very complex and interesting topic, we refer to the literature $[56,57]$. In the following we will first focus on different interfaces occurring in PHJ geometries and afterwards turn to effects observed in mixtures employed in BHJ cells.

\subsubsection{Interface in PHJ geometries}

As shown in Fig. 4.5a-c the interface in PHJ can have different geometries. Obviously, the schematics represent only ideal cases. In real thin films, the interfaces are usually not as well defined and even the distinction between PHJ and BHJ is often not as clear as depicted in the Fig. 4.5.

The growth of an organic material on top of another one can be different to the compared growth observed of this material on a bare substrate [58]. The growth of the overlayer can strongly depend on the film properties of the materials underneath. The interactions between different organic materials might be drastically different compared to the ones between a bare substrate and an organic compound and can have a strong influence on the growth [31]. These interactions depend inter alia on the type of energetic arrangement, i.e. whether dipole interactions or higher-order electronic interactions dominate the van-der-Waals interaction, on the sterical compatibility of the materials, which can lead to strain in the growth of the overlayer, and on the orientation of the bottom material, e.g. lying-down or standing-up orientation of rod-like molecules. 

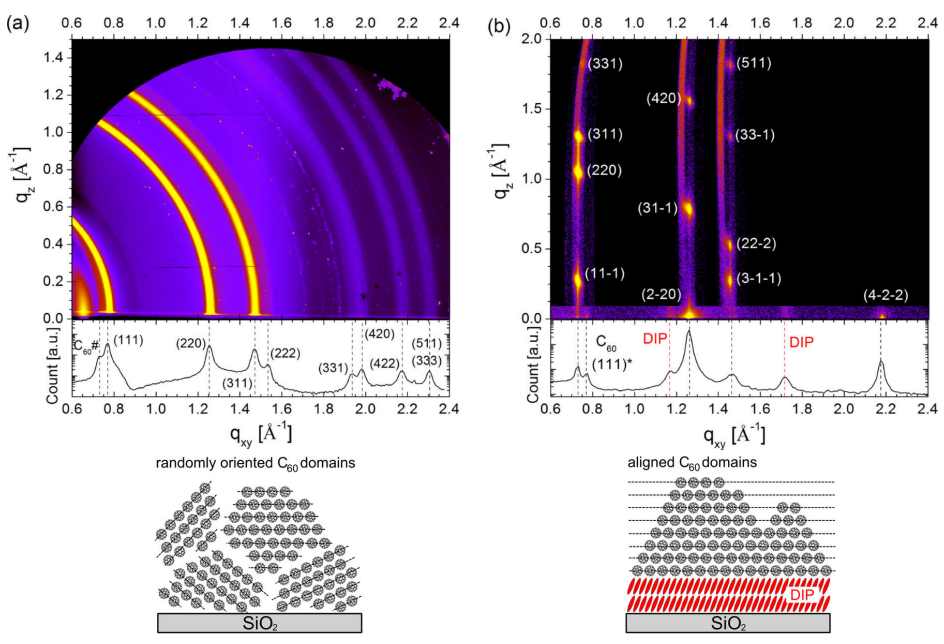

Fig. 4.11. (a) Reciprocal space map from a $60 \mathrm{~nm}$ C60 film recorded with a MARCCD area detector. At the bottom additional GIXD data measured with a point detector at $q_{z}=0.02 \AA^{-1}$ are shown. (b) 2-Dimensional GIXD data from a $15 \mathrm{~nm}$ C60 film grown on a DIP templating layer $(\mathrm{d}=4 \mathrm{~nm})$ indexed according to the C60 fcc structure. The data consist of four detector scans at a fixed angle of incidence at $0.1^{\circ}$ performed with a PILATUS II area detector. Images from each data point were transformed into $q$ coordinates and then assembled into one image. One scan was performed parallel to the substrate plane, for which at the bottom the integrated GIXD data are shown. Three scans were performed along the C60 crystal truncation rods. The schematics below show the growth of C60 on bare $\mathrm{SiO} 2$ and on a DIP templating layer, respectively. The figure is adapted from [58].

As an example, we show how real-time XRR can be used to characterize the growth of an organic heterostructure consisting of a PFP overlayer on top of DIP [55]. From the real-time data the roughness evolution of the film was extracted (Fig. 4.10). The PFP is first filling the voids between the DIP islands leading to a smoothing of the DIP layer. The resulting structure is very similar to the interface sketched in Fig. $4.5 \mathrm{~b}$.

The growth of an organic molecule on top of another can be different to the growth on a bare substrate. The structural order of C60 thin films is shown to be significantly improved by inserting a templating layer of DIP between the $\mathrm{SiO} 2$ substrate and $\mathrm{C} 60$. In contrast to growth on an amorphous substrate like $\mathrm{SiO} 2, \mathrm{C} 60$ grown on DIP exhibits alignment of fcc-domains with the (111) plane parallel to the substrate and a significant increase of the coherent in-plane island size by a factor of $\sim 4$ (Fig. 4.11) [58].

\subsubsection{Phase separation in BHJ geometries}

Mixtures of at least two organic compounds show interesting effects during the growth of thin films. The mixing scenario of two organic compounds depends 


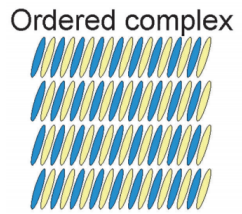

$\chi<0$

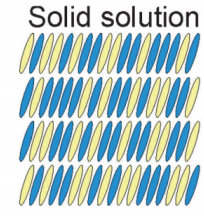

$\chi \approx 0$

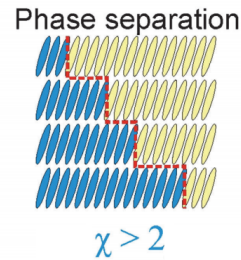

$\chi>2$

Fig. 4.12. Sketch of the different mixing scenarios usually observed in binary mixtures of organic semiconductors. Depending on the intermixing parameter $\chi$, either a mixed crystal, a solid solution or phase separation can occur. The figure is adapted from [31].

on several aspects, inter alia on the sterical compatibility of the two materials, the interaction energies between the two materials and between the materials and the substrate, and also on the preparation conditions, such as substrate temperature and deposition rate. According to Refs. [31, 59] the free energy of a mixture following a mean-field approach [60] can be written as:

$$
\frac{F_{m i x}}{k_{B} T}=x_{A} \ln x_{A}+x_{B} \ln x_{B}+\chi x_{A} x_{B}
$$

Here $x_{A}$ and $x_{B}$ are the concentrations of the two materials and $\chi$ is a dimensionless interaction parameter depending on the interaction energies between molecules of the same species $W_{A A}, W_{B B}$ and of the different species $W_{A B}$, respectively, and can be expressed as:

$$
\chi=\frac{1}{k_{B} T}\left[W_{A A}+W_{B B}-2 W_{A B}\right]
$$

Please note that this equation does not directly depend on the shape and dimensions, so the sterical compatibility, of the two molecules. As a guideline, for a good intermixability sterical compatibility can be assumed to be necessary. Depending on the value of the interaction parameter $\chi$, three different mixing scenarios can occur (see Fig. 4.12):

- Solid solution: If the interaction energies between the two species is similar to the ones within one species $(e . g . \chi \approx 0)$ the formation of a solid solution is favoured. So in this case a molecule of one species can be substituted by one of the other species. Such a mixing can usually be observed in systems of compounds with a resembling shape and an energetic similar arrangement, like PEN and DIP [61].

- Phase separation: If the two materials are sterically incompatible [62] or the interaction energies within the single materials are significantly more attractive than the inter-species energy $(\chi>2)$ usually a phase separation of the materials is observed. 


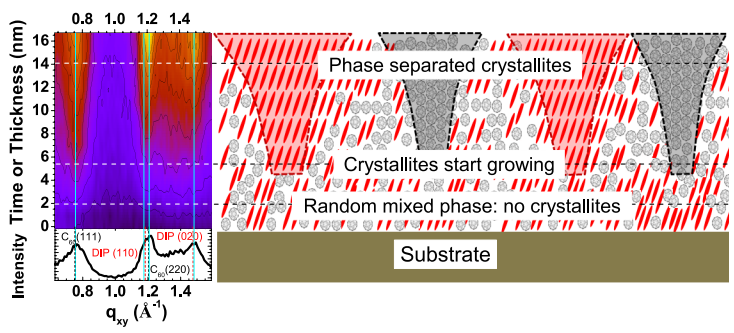

Fig. 4.13. The left hand side shows at the top real-time GIXD data from the film growth, indicating stronger and sharper peaks with increasing film thickness. On the right hand side a schematic of the cross-section parallel to the surface normal of a DIP:C60 mixture, showing first a random mixed phase and then the formation and following-up extension of domains of the pure materials is observed. Reprinted with permission [62].

- Ordered complex: An ordered complex is usually formed if the interaction energy between the two different materials is higher than the interaction energies within one species and the materials are sterically compatible $[61,63,64]$.

A prototypical phase separating system is a mixture of the two sterically incompatible materials DIP and C60. Real-time, in situ GIXD measurements revealed that in this system first a randomly mixed phase is formed and only after a certain film thickness the phase-separation begins [62]. With increasing film thickness the size of the crystalline domains and hence the magnitude of phase separation increases (Fig. 4.13). This effect shows that thin film growth is far away from equilibrium and the formation of the domains are kinetically limited. The resulting bottom-to-top asymmetry might have significant impact on the charge transport parallel, as well as perpendicular to the substrate surface.

\subsection{Exciton diffusion and dissociation}

Indicated in the introductory section, the exciton diffusion towards the separating DA interface constitutes an essential step in the power conversion of planar heterojunction solar cells. Therefore, the individual molecular layer thickness has to be optimized to guarantee a maximum absorption of incident light ( $\nearrow$ thickness) while simultaneously enabling photogenerated excitons to reach the DA junction within their lifetime $\tau$ ( $\searrow$ thickness). The one-dimensional diffusion relation $L_{\mathrm{D}}=\sqrt{D \tau}$ translates the latter into an effective exciton diffusion length $L_{\mathrm{D}}$. Accounting for the exponential thickness dependence of the absorption (Lambert-Beer law) and the one-dimensional diffusion behaviour (Fick's law), the mutual relation of the different contributions and their variation with layer thickness are illustrated in Fig. 4.14a. 
Whereas spectral absorption is mainly determined by the individual molecules and their next-neighbouring coupling in (poly-)crystalline thin films, the exciton diffusion length $L_{\mathrm{D}}$ shows a pronounced dependence on the respective film morphology, in particular, on the crystalline phase stabilized for a given substrate and its structural coherence length [66]. The susceptibility of the exciton transport on the underlying morphology becomes immediately obvious considering the broad variety of $L_{\mathrm{D}}$ values reported in literature, even for the same molecular compound on similar substrates [67].

Hence, a key challenge to access the inherent mechanisms governing exciton transport and its influence by structural or chemical inhomogeneities is the reliable determination of this property on well-defined molecular reference systems. Besides various techniques, photoluminescence (PL) quenching measurements facilitate a straight approach on this parameter and, by complementary structural information and time-resolved spectroscopy, establish its relation to sample morphology and excitonic state dynamics, respectively. The general concept of this technique is highlighted in Fig. 4.14 and has been discussed in detail in literature $[67,68]$. An exciton transport layer of defined thickness is partially covered by a quenching material, which by its energy level positions allows for quenching of the excitons reaching the interface. This effect is visualized in Fig. 4.14c for a wedged diindenoperylene layer of 100 to $400 \mathrm{~nm}$ thickness, capped by a $10 \mathrm{~nm}$ thick copper phthalocyanine $(\mathrm{CuPc})$ quencher layer (see Fig. 4.2). Apparently, with increasing DIP film thickness the PL intensities measured on the bare and $\mathrm{CuPc}$-covered areas resemble each other, indicating that excitons are no longer able to reach the $\mathrm{DIP} / \mathrm{CuPc}$ quencher interface. This quenching can appear either by a complete exciton transfer,

a)

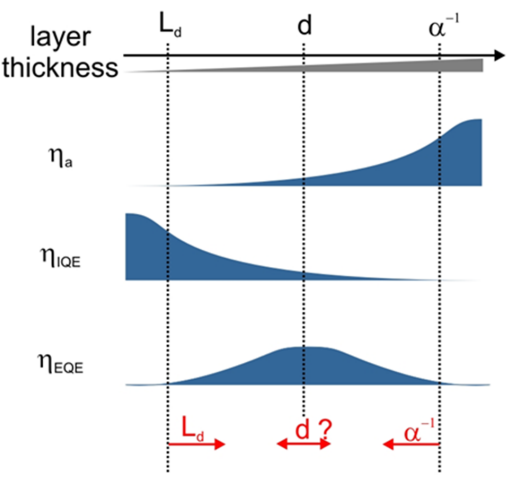

b)

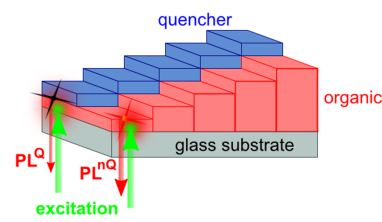

c)

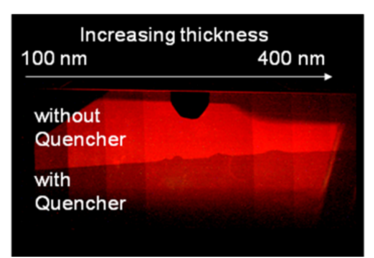

Fig. 4.14. (a) Thickness dependence of absorption depth, $\alpha^{-1}$, and exciton diffusion length $L_{\mathrm{D}}$, determining the optimum film thickness, $d$, of stacked photovoltaic cells. (b) Schematic of the photoluminescence (PL) quenching measurements. Adapted from [65]. (c) Photograph of the thickness dependent PL of DIP with and without $\mathrm{CuPc}$ quenching layer atop. With increasing DIP thickness, less excitons are able to reach the DIP/CuPc interface within their lifetime, effectively increasing the PL. 
e.g. by Förster or Dexter mechanism as in the case of $\mathrm{DIP} / \mathrm{CuPc}$ [65], or by converting the Frenkel-type exciton into a long-lived charge transfer (CT) state or free charge carriers as for $\mathrm{DIP} / \mathrm{C} 60$ or $\mathrm{ZnPc} / \mathrm{C} 60[8,69]$. As a figure of merit, the relative quenching $Q$ defined by the PL intensity ratio with and without quencher

$$
Q=\frac{I_{\mathrm{PL}, \mathrm{Q}}}{I_{\mathrm{PL}, \mathrm{nQ}}}
$$

can be utilized and provides detailed information on the diffusion length and quenching quality at the quencher interface. In addition, more elaborated models can account for interference effects by the incident light as well as for the microscopic morphology of real interfaces [65,70]. For many molecular semiconductors growth on weakly interacting substrates such as ITO/PEDT:PSS or $\mathrm{MoO}_{3}$ proceeds via island formation (Vollmer-Weber mode) leading to a pronounced interface roughness as indicated in Fig. 4.10. In case of non-perfectly smooth interfaces in long-range ordered crystalline films, i.e. where the exciton diffusion length might reach the absorption length, care has to be taken on a comprehensive modeling as the well-established FengGhosh model [71] in its initial form is not applicable.

Representative PL quenching studies on prototypical molecular semiconductors are displayed in Fig. 4.15 together with the structural properties as determined by X-ray diffraction. Despite of the X-ray amorphous film structure, thickness dependent PL quenching measurements on (tris(8-hydroxyquinoline)aluminum (Alq3) revealed an exciton diffusion length of about $22 \mathrm{~nm}$ at room temperature exceeding that of many disordered organic materials by far [67]. Remarkably, a similar trend has been observed for C60 thin films $\left(L_{\mathrm{D}}=25 \mathrm{~nm}\right)$, indicating a support of exciton motion by the spherical shape of the molecules via dense packing of the homogeneously distributed transition dipoles.

In contrast, DIP and $6 \mathrm{~T}$ layers show a pronounced crystalline ordering in the X-ray diffraction spectra which coincides with an exceptional singlet diffusion lengths of about $90 \mathrm{~nm}$ for both compounds at room temperature (see Fig. 4.15b and c). In analogy to charge carrier motion, this might be related to a distinct wavefunction overlap along preferred directions of the crystalline lattice. However, as it turned-out by comprehensive time-dependent DFTcalculations (SCS-CC2 method) on a dimer level, the relative motion between adjacent molecules upon electronic excitation is essential for supporting or impeding exciton diffusion $[72,73]$. Depending on the respective unit cell packing, this relative motion can lead to a continuous downward shift of the excited state on the related potential energy landscape (PEL) until a conical intersection with the PEL of an energetically lower-lying state is reached and the exciton is efficiently transferred to this dark state within its lifetime. Typically, this processes occurs within several hundred femtoseconds after photoexcitation and thus defines an intrinsic localization time [73]. Whereas for many 
a)

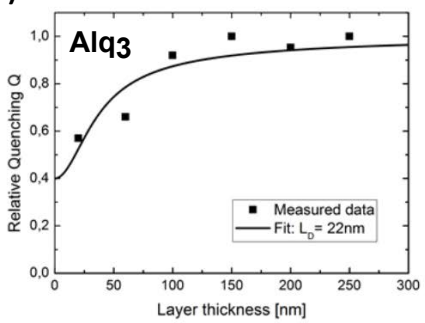

c)

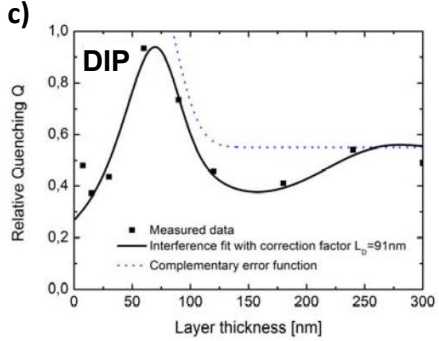

b)

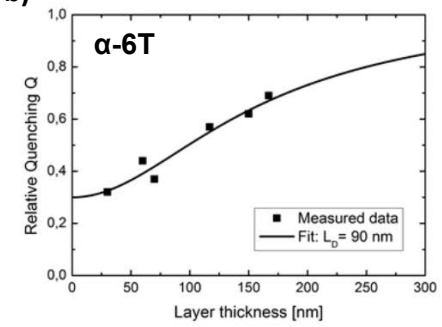

d)

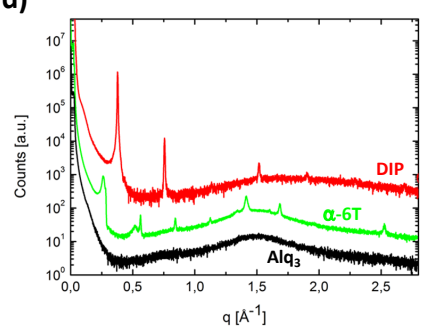

Fig. 4.15. (a)-(c) Measured and simulated PL quenching on various, prototypical molecular semiconductors. (d) X-ray diffraction analyses of the corresponding thin film structures evidencing the long range ordering in case of DIP and $6 \mathrm{~T}$ and the X-ray amorphous film structure of Alq3.

materials, including perylene-derivatives, this process leads to rather short exciton diffusion lengths of $22 \mathrm{~nm}$ for perylenetetracarboxylic dianhydride $(\alpha-$ PTCDA) in agreement with literature [66], the situation differs remarkably in case of DIP. Due to the specific alignment of molecular pairs within the thin film unit cell, the inter-molecular motion upon electronic excitation imposes an energy barrier on the related energy manifold which prevents excitons from reaching the conical intersection and thereby, extends their effective diffusion length. As a consequence of this steric hindrance $L_{\mathrm{D}}$ is mainly governed by the radiative-lifetime of the excited state and the spatial extension of crystalline grains. This was evidenced by temperature dependent PL quenching studies on DIP as well as $6 \mathrm{~T}$ rendering exciton transport to be non-thermally activated below a critical temperature and film thickness. This observation was explained by a reduced exciton-phonon scattering at low temperatures and hints at a coherent exction motion within the crystalline grains [65]. Furthermore, in such long-range ordered crystalline systems efficient exciton trapping might occur at grain boundaries where the structural constraints are lifted as reported for rubrene microcrystals [74].

Motivated by the enhanced exciton diffusion length observed in DIP various derivatives have been investigated and are shown with respect to their thickness dependent PL quenching behaviour in Fig. 4.16. According to simulations, the related exciton diffusion lengths amount to 5, 13 and $25 \mathrm{~nm}$ for 

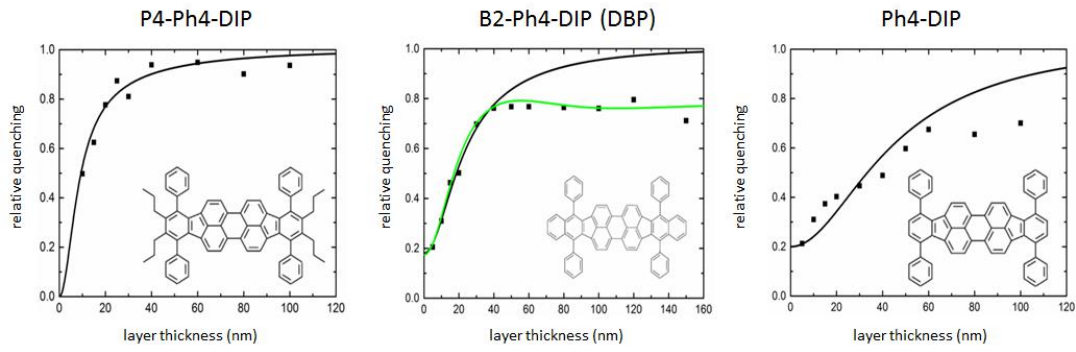

Fig. 4.16. Relative PL quenching measurements on various DIP derivatives (provided by courtesy of IAPP, TU-Dresden). The respective exciton diffusion lengths amount to 5, 13 and $25 \mathrm{~nm}$ for P4-PH-DIP, B2-Ph4-DIP (DBP) and Ph4-DIP, respectively. All layers form an X-ray amorphous film structure.

tetrapropyltetraphenyldiindenoperylene (P4-Ph4-DIP), DBP (B2-Ph4-DIP) and tetraphenyldiindenoperylene (Ph4-DIP), respectively. Moreover, as can be concluded from the constant quenching offset for B2-Ph4-DIP and Ph4DIP thicknesses above $50 \mathrm{~nm}$ a roughening of the films occurs which constitutes a precondition for a self-organized interdigitated film structure, the latter considered to be preferential for planar heterojunction solar cells (see Fig. 4.5).

Even though, owing to the amorphous film structure upon deposition on unheated substrates, the diffusion lengths in these samples are inferior to that of long-range ordered DIP $\left(L_{\mathrm{D}}=90 \mathrm{~nm}\right)$, this disadvantage is partially compensated by the much better absorption characteristics due to the randomly distributed transition dipole moments (see Fig. 4.4). Hence, the overall power conversion efficiencies for planar Ph4-DIP/C60 or bulk Ph4-DIP:C60 heterojunction cells resemble that of the corresponding DIP devices, but with the additional possibility of further enhancing the crystalline order by e.g. thermal treatment [75].

Finally, addressing the non-radiative losses by exciton-polaron interaction in thin film solar cells a new approach has been proposed, allowing not only for discrimination between effects by free and localized, i.e. trapped, charge carriers but also for distinction between electrons and holes. For this purpose, standard thin film transistor (TFT) geometry has been employed, enabling photogenerated excitons to interact with an adjustable amount of charge carriers within a spatially well-defined sample region of monolayer thickness. The general principle of this method is illustrated in Fig. 4.17a and relies on the voltage controlled accumulation of the desired charge carrier density at the boundary between the organic transport layer and a chemically passivated gate dielectric.

Applying this technique on crystalline DIP thin films, the relative quenching $Q$, defined by the $\mathrm{PL}$ intensity ratio at various gate-voltages $V_{\mathrm{G}}$ with respect to that at $V_{\mathrm{G}}=0 \mathrm{~V}$ is measured and shown in Fig. 4.17b. A distinct 
a)

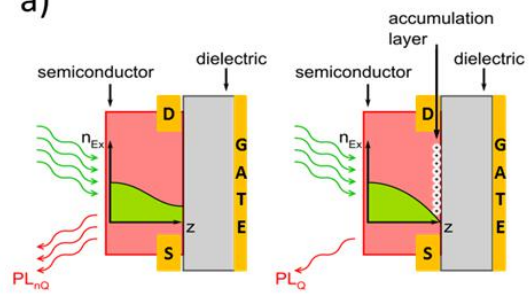

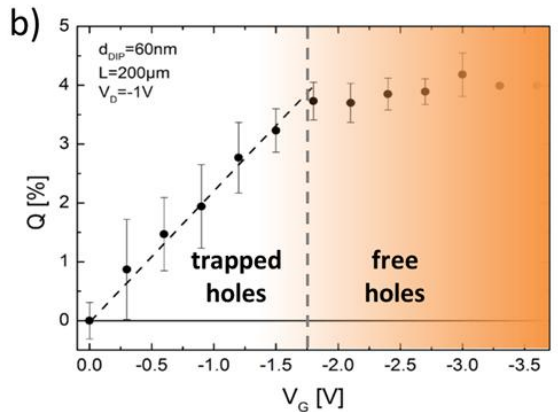

Fig. 4.17. a) Principle of TFT-assisted determination of loss mechanisms by exciton-polaron interaction. b) Relative PL quenching $Q$ at different gate voltages, indicating the impact of trapped and free charge carriers on non-radiative exciton losses. Adapted from [76].

linear variation of the relative quenching occurs up to a certain threshold voltage of about $-1.7 \mathrm{~V}$, whereas above this value, the quenching remains almost constant. According to TFT theory, this low voltage regime can be attributed to gradual trap filling at the interface of the organic transport layer by injected holes (negative gate voltage), while the high voltage regime is assigned to free charge carriers within the conduction channel. Therefore, the exciton losses, indicated by an increase of the relative quenching can be related to the interaction with localized polarons rather than with free holes. Moreover, the maximum quenching of about $4.5 \%$ indicates the minor importance of non-radiative recombination losses in crystalline DIP thin films, as has been confirmed by spectrally resolved photocurrent measurements. Accordingly, the recombination rate of non-radiative exciton-hole processes can be estimated to $1.1 \times 10^{-12} \mathrm{~cm}^{3} / \mathrm{s}[76,77]$, evidencing the potential of the described approach for identifying the microscopic mechanisms of exciton-charge carrier interaction as well as quantifying their respective cross-sections.

Based on the experimental findings and theoretical results general strategies towards a rational design of molecular compounds can be developed, yielding to further improvement of existing device concepts by an optimized planar heterojunction architecture with respect to exciton generation and transport.

\subsection{Charge transport and device performance}

Solar cells have been investigated for various DA combinations shown in Figs. 4.2, 4.3 and 4.4 (see Refs. [12, 25,36,78-80]). Among them DIP/C60 turned out to be a model system, because in depth studies of electronic structure, morphology and exciton transport were available. Remarkably, DIP grows in a crystalline fashion on many technical substrates and, in particular, on ITO covered with PEDT:PSS or HIL1.3 used as anode for OPVCs in this study. 

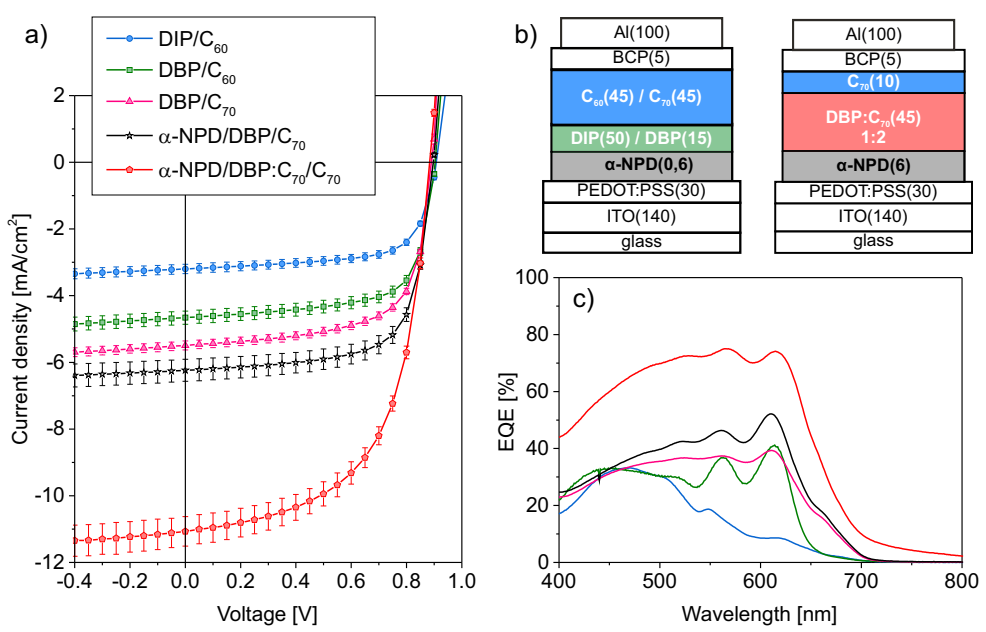

Fig. 4.18. (a) Comparison of $J-V$ characteristics under simulated AM1.5g illumination of PHJ and PM-HJ devices with DIP and DBP as donors and C60 as well as C70 as acceptors, together with (b) photocurrent spectra of the same devices. The inset shows the schematic layer structure of the cell. Four nominally identical pixels with an active area of $4 \mathrm{~mm}^{2}$ each were placed on a $20 \mathrm{~mm} \times 20 \mathrm{~mm}$ substrate with a common ITO electrode. $J-V$ measurements were performed without masking the illuminated pixel; for the photocurrent spectra the illumination spot was smaller than the pixel size. By comparison of both values - the directly measured $J_{\mathrm{SC}}$ and the spectrally integrated photocurrent - we obtained consistent values. The figure is adapted from [25].

Even without substrate heating the molecules form crystalline domains with sizes of a few $10 \mathrm{~nm}$ and for heated substrates (with $T_{\mathrm{S}} \approx 100^{\circ} \mathrm{C}$ ) the lateral domain size reaches almost micron dimensions. This high degree of structural order is reflected in good charge transport properties of DIP reaching carrier mobilities of $10^{-1} \mathrm{~cm}^{2} / \mathrm{Vs}$ for holes as well as electrons in field-effect transistors [81]. This ambipolar transport behaviour together with its energy levels being intermediate between many classical donor and acceptor materials (see Fig. 4.3) make DIP a unique candidate for implementation as both donor or acceptor, depending on the partner material [78]. On the one hand, it is a donor with respect to fullerenes, however, with significantly larger $V_{\mathrm{OC}}$ than $\mathrm{CuPc}, 6 \mathrm{~T}$ or PEN. On the other hand, it can also be used as an acceptor for $6 \mathrm{~T}$ and its polymeric analogue $\mathrm{P} 3 \mathrm{HT}$, yielding $V_{\mathrm{OC}}$ of more than $1.3 \mathrm{~V}$.

Figure 4.18 shows photoelectrical characteristics of DIP and its related analogue DBP combined with C60 or C70 in different architectures. DIP/C60 cells yield a high open circuit voltage exceeding $0.9 \mathrm{~V}$ and high fill factors (up to $75 \%$ ), however, they lack in current because owing to the uprightstanding DIP growth there is basically no photon absorption and therefore no significant contribution of DIP to the photocurrent (see Fig. 4.18c). This 
Table 4.1. Summary of device data for DIP/C60 PHJs and DBP:C70 PM-HJs under simulated AM1.5g illumination. The values were averaged over four nominally identical pixels on the same substrate. An exemplary $J-V$ curve for each type is shown in Fig. 4.18.

\begin{tabular}{ccc}
\hline & DIP $/ \mathrm{C} 60$ & $\alpha$-NPD/DBP:C70/C70 \\
\hline$j_{\mathrm{SC}}\left[\mathrm{mA} / \mathrm{cm}^{2}\right]$ & $3.16 \pm 0.18$ & $11.07 \pm 0.44$ \\
$V_{\mathrm{OC}}[\mathrm{mV}]$ & $916 \pm 1$ & $884 \pm 1$ \\
$F F[\%]$ & $65.8 \pm 0.9$ & $58.9 \pm 0.7$ \\
$\eta_{\mathrm{PCE}}[\%]$ & $1.90 \pm 0.03$ & $5.76 \pm 0.19$ \\
\hline
\end{tabular}

deficit can be improved by using DBP with lying orientation as donor material. As the EQE spectra clearly show, there is now an equivalent contribution of both materials. Further improvement of $J_{\mathrm{SC}}$ is possible, if the fullerene C70 is used as acceptor, which has higher absorption than C60 due to the lower symmetry of the molecule. Nevertheless, as the photocurrent spectra show, part of the improvement comes at the expense of less photocurrent from DBP. The latter can be enhanced, if a thin layer of $\alpha$-NPD is introduced as an exciton blocking layer to prevent quenching at the interface to the highly conducting PEDT:PSS. The biggest increase in short-circuit current, however, is achieved when the PHJ architecture is replaced by a PM-HJ - in this case DBP mixed with C70 in a 1:2 ratio by weight. As Fig. 4.18 shows the current almost doubles and an EQE between 70 and $80 \%$ is achieved over a wide range. From the point of view of exciton quenching, the $\alpha$-NPD layer is no longer required, but it is necessary to keep the effective work function of the anode high enough to maintain the high open circuit voltage. Overall, in going from PHJ of DIP/C60 to PM-HJ with DBP and C70 the power conversion efficiency has been increased from about $2 \%$ to almost $6 \%$ (see Table 4.1 ).

To understand the origin and magnitude of the open circuit voltage in OPVCs, we performed detailed studies of the temperature dependent electrical device characteristics (see also Ref. [79, 80, 82]). As an example, Fig. 4.19 shows $J-V$ characteristics of a DIP/C60 PHJ device measured in dark and under different illumination conditions. In general, under white light illumination (with intensity comparable to AM1.5g, Fig. 4.19a) one observes an increase of $V_{\mathrm{OC}}$ upon cooling, which is accompanied by a decrease in photocurrent and the formation of a so-called S-shape. Both of these unwanted effects are related to thermally activated injection and transport of carriers. The dark and light characteristics can be described with a modified Shockley-Queisser model as explained in Ref. [82]. The description is based on a simplified absorption spectrum displayed in Fig. 4.19c, where in addition to the fundamental absorption across the optical gap at $E_{\mathrm{G}}$ a second absorption step at the CT energy $E_{\mathrm{CT}}$ with an absorption strength $\alpha_{\mathrm{CT}}$ is introduced. According to this model the temperature dependence of $V_{\mathrm{OC}}$ follows a relation $[7,83]$ : 

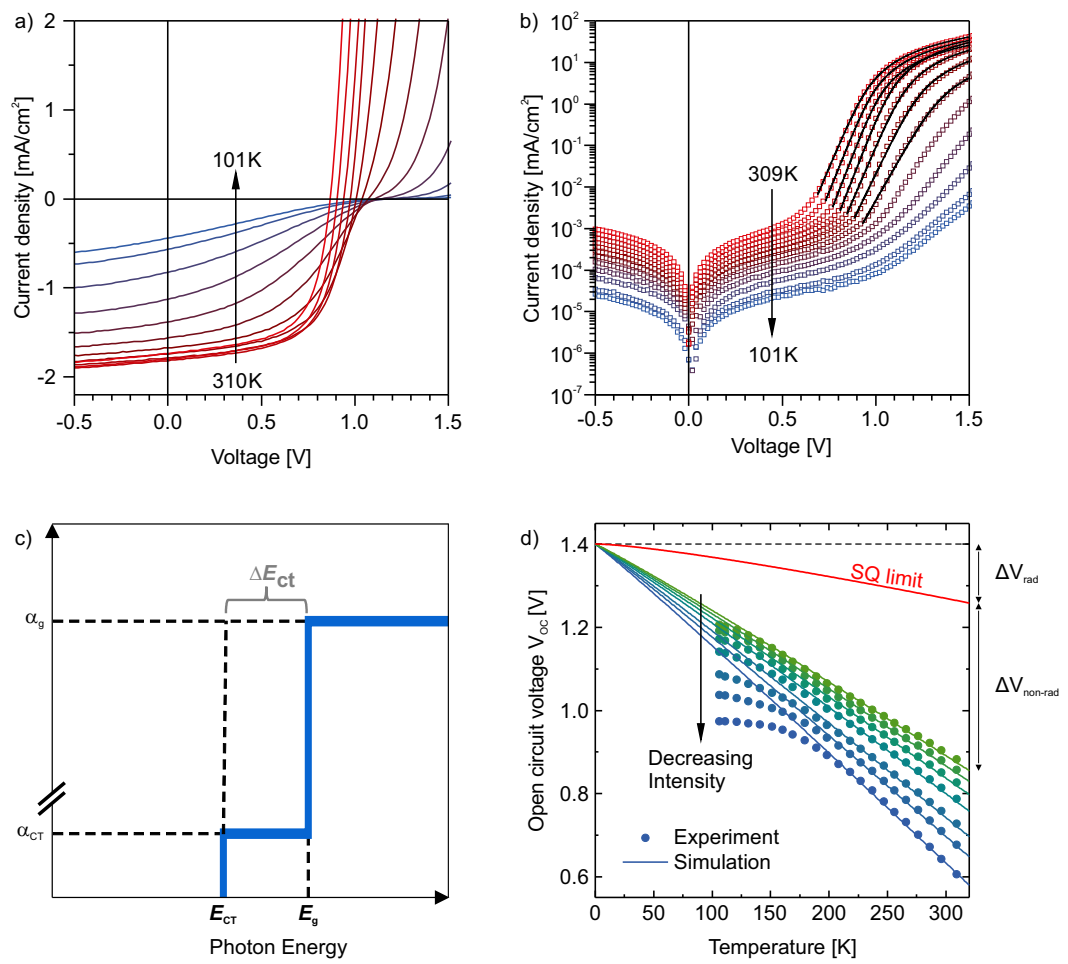

Fig. 4.19. Temperature dependent $J-V$ characteristics for a DIP/C60 PHJ in the dark (a) and under white light illumination (b). Part (d) shows the temperature dependence of the $V_{\mathrm{OC}}$ under different illumination intensities together with simulations according to a modified SQ limit, where the schematic absorption spectrum displayed in (c) was used. The temperature dependent $V_{\mathrm{OC}}$ data are well described assuming a CT energy $E_{\mathrm{CT}}=1.4 \mathrm{eV}$, an absorption strength of the CT state of $\alpha_{\mathrm{CT}}=1.5 \times 10^{-4}$ (relative to the main absorption $\alpha_{0}$ at $E_{\mathrm{opt}}=1.9 \mathrm{eV}$ ) and assuming non-radiative recombination to be higher by a factor $\beta=2 \times 10^{6}$ than the radiative one. The figure is adapted from [79].

$$
V_{\mathrm{OC}}=\frac{E_{\mathrm{CT}}}{e}-\Delta V_{\mathrm{rad}}-\Delta V_{\mathrm{non}-\mathrm{rad}},
$$

where both of the radiative and non-radiative loss terms depend approximately linearly on temperature [79]. Thus, from the temperature and intensity dependent $V_{\mathrm{OC}}$ data shown in Fig. 4.19d one obtains a CT energy of $1.4 \mathrm{eV}$, which sets the upper limit for the chemical potential of photogenerated charge carriers at $T=0 \mathrm{~K}$. Additionally, the graph shows that the major loss at room temperature is due to non-radiative recombination $\left(\Delta V_{\text {non-rad }} \approx 0.4 \mathrm{~V}\right)-$ in agreement with the non-radiative recombination current being more than six orders of magnitude higher than the radiative one, whereas the thermodynamically unavoidable radiative recombination across the CT gap amounts to 

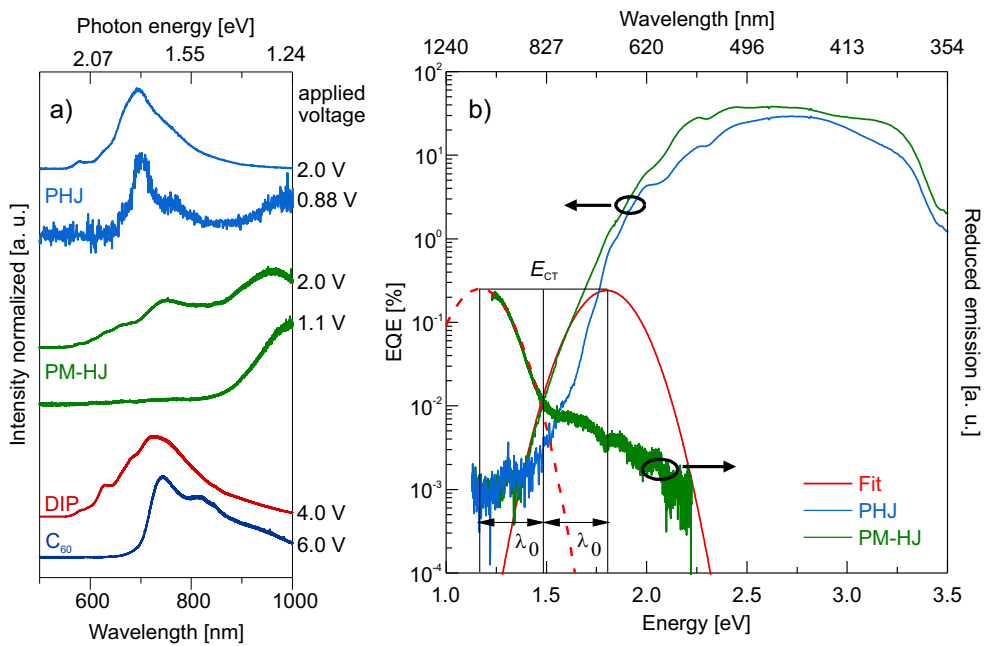

Fig. 4.20. (a) Electroluminescence spectra of single layer diodes with DIP and C60 as active layers together with the spectra of PHJ and PM-HJ structures of both materials clearly showing an additional emission band in the near infrared, where none of the two neat materials shows luminescence. (b) Reduced electroluminescence and sensitively measured EQE spectra in the range of the CT state absorption and emission. From Gaussian fits with identical reorganization energy $\lambda_{0} \approx 0.3 \mathrm{eV}$ a CT energy of $1.45 \mathrm{eV}$ is obtained.

a loss $\Delta V_{\text {rad }}$ of only $0.1 \mathrm{~V}$. This exemplifies that the energy loss between the $\mathrm{CT}$ state and the open circuit voltage is of the order of $0.5 \mathrm{eV}$ and, thus, in the same range as in many polymer:fullerene OPVCs [7]. However, one has to keep in mind that there is an additional energy loss occurring in the formation of the CT state in the course of exciton dissociation at the DA interface (see Fig. 4.1).

The apparently linear extrapolation of the measured $V_{\mathrm{OC}}(T)$ towards $T=0 \mathrm{~K}$ also deserves some comment. After introduction by Vandewal et al. [7], this approach to determine the relevant photovoltaic gap has been frequently used in the literature $[82,84]$. However, as we have found with the system $6 \mathrm{~T} / \mathrm{DIP}$, it can overestimate the actual CT energy at the DA interface, if the CT gap is close to the optical gap of one of the two materials and if the electronic coupling between both of them is extremely weak, as is the case for the standing orientation of both molecules on top of each other [80]. In this case, a linear extrapolation of measured $V_{\mathrm{OC}}(T)$ data yields the optical gap of DIP, which indicates that this is the dominant recombination pathway at higher temperatures.

Direct spectroscopic access to CT states in OPVCs can be obtained from electroluminescence (EL) and photocurrent spectra measured with high sensitivity below the optical gap of the main absorber [85]. Figure 4.20a shows EL spectra of diodes with neat layers of DIP and C60 each, which are com- 
pared to EL spectra of PHJ and PM-HJ of both materials, where the OPVCs are operated as light-emitting devices in the forward bias regime. For both DA heterojunctions one observes a broad emission band in the near infrared region $(850-1000 \mathrm{~nm})$, where none of the neat materials shows luminescence. In particular for the PM-HJ, the EL measured close to $V_{\mathrm{OC}}$ stems exclusively from light emission from $\mathrm{CT}$ states. A quantitative analysis combining the reduced emission spectrum with the EQE spectrum as shown in Fig. 4.20b reveals a $\mathrm{CT}$ energy of $1.45 \mathrm{eV}$, in excellent agreement with temperature dependent electrical measurements discussed above. What the spectra also show is a fairly large Stokes shift between CT absorption (modelled as a Gaussian in the low-energy onset of the EQE spectrum) and CT emission, being reflected in a shift between both maxima of twice the reorganization energy $\lambda_{0} \approx 0.3 \mathrm{eV}$. This large shift together with the low EL quantum efficiency can be seen as major sources of the relatively large energy losses in OPVCs as compared to e.g. hybrid inorganic-organic perovskite cells [86]. Understanding the detailed role of CT states in the process of charge generation in OPVCs, however, is subject of ongoing research.

\subsection{Conclusions}

The concerted studies over recent years have revealed important design rules for improving organic solar cell performance. Using well-defined model systems of molecular donor-acceptor pairs, we have been able to understand their growth behaviour in heterostructures with different architecture and, thus, to a certain extend tune their nanomorphology towards favourable conditions

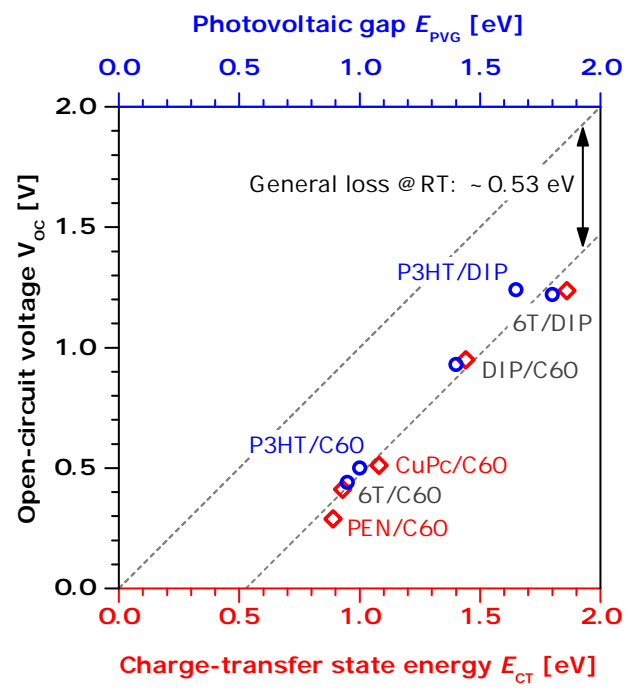

Fig. 4.21. Comparison of open circuit voltage dependence on charge transfer energy (red diamonds) and photovoltaic gap (blue circles). The difference for materials pairs analysed with both methods (grey labels) are within the uncertainties due to variations of material batches and sample preparation. The values for $V_{\mathrm{OC}}$ and $E_{\mathrm{CT}}$ are measured on the same sample [79]. For the other set $V_{\mathrm{OC}}$ is taken from literature $[78,80]$ in order to show small fluctuations between experimental runs. 
for OPVCs. This is reflected e.g. in highly crystalline layers allowing coherent exciton transport with diffusion lengths being only limited by the film thickness. The interface energetics has been studied for all relevant interfaces under device-relevant conditions and was correlated to solar cell performance data.

As a synopsis, Fig. 4.21 shows a compilation of the open circuit voltages of all investigated DA combinations with CT energies determined from temperature dependent photoelectrical characterization and the relevant photovoltaic gap from photoelectron spectroscopy. In both cases a linear dependence of $V_{\mathrm{OC}}$ on the respective interfacial gap is observed, yielding the so-called bandgapvoltage offset of $0.53 \mathrm{eV}$. As compared to most inorganic pn-junction solar cells [87] as well as the emerging perovskite cells [86] this offset is significantly larger and one of the reasons for lower performance of OPVCs. Future research will therefore, inter alia, have to find strategies to reduce energy losses in organic photovoltaics.

\section{References}

1. http://www.nrel.gov/ncpv/ (2015). (accessed on September 9th)

2. M.A. Green, K. Emery, Y. Hishikawa, W. Warta, E.D. Dunlop, Prog. Photovolt: Res. Appl. 23, 805812 (2015). DOI 10.1002/pip.2637

3. W. Shockley, H.J. Queisser, J. Appl. Phys. 32, 510 (1961). DOI 10.1063/1. 1736034

4. D. Veldman, S.C.J. Meskers, R.A.J. Janssen, Adv. Funct. Mater. 19, 1939 (2009). DOI 10.1002/adfm.200900090

5. T.M. Clarke, J.R. Durrant, Chem. Rev. 110, 6736 (2010). DOI 10.1021/ cr900271s

6. C. Deibel, V. Dyakonov, Rep. Prog. Phys. 73, 096401 (2010). DOI 10.1088/ 0034-4885/73/9/096401

7. K. Vandewal, K. Tvingstedt, A. Gadisa, O. Inganäs, J.V. Manca, Phys. Rev. B 81, 125204 (2010). DOI 10.1103/PhysRevB.81.125204

8. A. Opitz, J. Wagner, W. Brütting, I. Salzmann, N. Koch, J. Manara, J. Pflaum, A. Hinderhofer, F. Schreiber, IEEE J. Sel. Top. Quant. 16, 1707 (2010). DOI 10.1109/JSTQE.2010.2048096

9. S.R. Forrest, MRS Bull. 30, 28 (2011). DOI 10.1557/mrs2005.5

10. A. Wilke, T. Mizokuro, R.P. Blum, J.P. Rabe, N. Koch, IEEE J. Sel. Top. Quant. 16, 1732 (2010). DOI 10.1109/JSTQE.2010.2042035

11. I. Salzmann, S. Duhm, G. Heimel, M. Oehzelt, R. Kniprath, R.L. Johnson, J.P. Rabe, N. Koch, J. Am. Chem. Soc. 130, 12870 (2008). DOI 10.1021/ja804793a

12. J. Wagner, M. Gruber, A. Hinderhofer, A. Wilke, B. Bröker, J. Frisch, P. Amsalem, A. Vollmer, A. Opitz, N. Koch, F. Schreiber, W. Brütting, Adv. Func. Mater. 20, 4295 (2010). DOI 10.1002/adfm.201001028

13. S. Pfützner, Studies on organic solar cells composed of fullerenes and zinc-phthalocyanines. Ph.D. thesis, TU Dresden (2012). URL http:// nbn-resolving.de/urn:nbn:de:bsz:14-qucosa-83486

14. A. Opitz, J. Frisch, R. Schlesinger, A. Wilke, N. Koch, J. Electron. Spectrosc. Relat. Phenom. 190, 12 (2013). DOI 10.1016/j.elspec.2012.11.008 
15. K.M. Lau, J.X. Tang, H.Y. Sun, C.S. Lee, S.T. Lee, D. Yan, Appl. Phys. Lett. 88, 173513 (2006). DOI 10.1063/1.2198484

16. S. Krause, M.B. Casu, A. Schöll, E. Umbach, New J. Phys. 10, 085001 (2008). DOI 10.1088/1367-2630/10/8/085001

17. A. Wilke, J. Endres, U. Hormann, J. Niederhausen, R. Schlesinger, J. Frisch, P. Amsalem, J. Wagner, M. Gruber, A. Opitz, A. Vollmer, W. Brütting, A. Kahn, N. Koch, Appl. Phys. Lett. 101, 233301 (2012). DOI 10.1063/1. 4769360

18. W. Han, H. Yoshida, N. Ueno, S. Kera, Appl. Phys. Lett. 103, 123303 (2013). DOI 10.1063/1.4821445

19. H. Brinkmann, C. Kelting, S. Makarov, O. Tsaryova, G. Schnurpfeil, D. Wöhrle, D. Schlettwein, phys. stat. sol. a 205, 409 (2008). DOI 10.1002/pssa.200723391

20. P.I. Djurovich, E.I. Mayo, S.R. Forrest, M.E. Thompson, Org. Electron. 10, 515 (2009). DOI 10.1016/j.orgel.2008.12.011

21. S.M. Sze, K.K. Ng, Physics of Semiconductor Devices, 3rd edn. (John Wiley \& Sons, Inc., 2007). DOI 10.1002/0470068329

22. B. Rand, D. Burk, S. Forrest, Phys. Rev. B 75, 115327 (2007). DOI 10.1103/ PhysRevB.75.115327

23. M. Riede, T. Mueller, W. Tress, R. Schueppel, K. Leo, Nanotechnology 19, 424001 (2008). DOI 10.1088/0957-4484/19/42/424001

24. T. Stübinger, W. Brütting, J. Appl. Phys. 90, 3632 (2001). DOI 10.1063/1. 1394920

25. S. Grob, M. Gruber, A.N. Bartynski, U. Hörmann, T. Linderl, M.E. Thompson, W. Brütting, Appl. Phys. Lett. 104, 213304 (2014). DOI 10.1063/1.4879839

26. C.W. Tang, Appl. Phys. Lett. 48, 183 (1986). DOI 10.1063/1.96937

27. S. Yu, C. Klimm, P. Schäfer, J.P. Rabe, B. Rech, N. Koch, Org. Electron. 12, 2180 (2011). DOI 10.1016/j.orgel.2011.09.021

28. S. Yu, A. Opitz, S. Grob, R. Resel, M. Oehzelt, W. Brütting, I. Salzmann, N. Koch, Org. Electron. 15, 2210 (2014). DOI 10.1016/j.orgel.2014.06.023

29. I. Salzmann, S. Duhm, R. Opitz, R.L. Johnson, J.P. Rabe, N. Koch, J. Appl. Phys. 104, 114518 (2008). DOI 10.1063/1.3040003

30. P. Peumans, S. Uchida, S.R. Forrest, Nature 425, 158 (2003). DOI 10.1038/ nature01949

31. A. Hinderhofer, F. Schreiber, ChemPhysChem 13, 628 (2012). DOI 10.1002/ cphc. 201100737

32. A. Opitz, B. Ecker, J. Wagner, A. Hinderhofer, F. Schreiber, J. Manara, J. Pflaum, W. Brütting, Org. Electron. 10, 1259 (2009). DOI 10.1016/j.orgel. 2009.07.004

33. A. Hinderhofer, C. Frank, T. Hosokai, A. Resta, A. Gerlach, F. Schreiber, J. Chem. Phys. 134, 104702 (2011). DOI 10.1063/1.3557476

34. J.P. Reinhardt, A. Hinderhofer, K. Broch, U. Heinemeyer, S. Kowarik, A. Vorobiev, A. Gerlach, F. Schreiber, J. Phys. Chem. C 116, 10917 (2012). DOI 10.1021/jp211947y

35. A. Opitz, J. Wagner, W. Brütting, A. Hinderhofer, F. Schreiber, phys. stat. sol. a 206, 2683 (2009). DOI 10.1002/pssa.200925238

36. M. Gruber, M. Rawolle, J. Wagner, D. Magerl, U. Hörmann, J. Perlich, S.V. Roth, A. Opitz, F. Schreiber, P. Müller-Buschbaum, W. Brütting, Adv. Energy Mater. 3, 1075 (2013). DOI 10.1002/aenm.201201012

37. A. Wilke, P. Amsalem, J. Frisch, B. Bröker, A. Vollmer, N. Koch, Appl. Phys. Lett. 98, 123304 (2011). DOI 10.1063/1.3571286 
38. H. Ishii, K. Sugiyama, E. Ito, K. Seki, Adv. Mater. 11, 605 (1999). DOI 10.1002/(SICI)1521-4095(199906)11:8<605::AID-ADMA605>3.0.CO;2-Q

39. D. Cahen, A. Kahn, Adv. Mater. 15, 271 (2003). DOI 10.1002/adma.200390065

40. N. Koch, ChemPhysChem 8, 1438 (2007). DOI 10.1002/cphc.200700177

41. N. Ueno, S. Kera, Prog. Surf. Sci. 83, 490 (2008). DOI 10.1016/j.progsurf.2008. 10.002

42. C. Wu, Y. Hirose, H. Sirringhaus, A. Kahn, Chem. Phys. Lett. 272, 43 (1997). DOI 10.1016/S0009-2614(97)00481-8

43. M. Oehzelt, N. Koch, G. Heimel, Nat. Commun. 5, 4174 (2014). DOI 10.1038/ ncomms5174

44. H. Wang, P. Amsalem, G. Heimel, I. Salzmann, N. Koch, M. Oehzelt, Adv. Mater. 26, 925 (2014). DOI 10.1002/adma.201303467

45. K. Akaike, N. Koch, M. Oehzelt, Appl. Phys. Lett. 105, 223303 (2014). DOI $10.1063 / 1.4903360$

46. J. Wagner, M. Gruber, A. Wilke, Y. Tanaka, K. Topczak, A. Steindamm, U. Hörmann, A. Opitz, Y. Nakayama, H. Ishii, J. Pflaum, N. Koch, W. Brütting, J. Appl. Phys. 111, 054509 (2012). DOI 10.1063/1.3692050

47. P. Amsalem, J. Niederhausen, A. Wilke, G. Heimel, R. Schlesinger, S. Winkler, A. Vollmer, J. Rabe, N. Koch, Phys. Rev. B 87, 035440 (2013). DOI 10.1103/ PhysRevB.87.035440

48. P. Peumans, S.R. Forrest, Appl. Phys. Lett. 79, 126 (2001). DOI 10.1063/1. 1384001

49. A. Steindamm, M. Brendel, A.K. Topczak, J. Pflaum, Appl. Phys. Lett. 101, 143302 (2012). DOI 10.1063/1.4757297

50. P. Heremans, D. Cheyns, B.P. Rand, Acc. Chem. Res. 42, 1740 (2009). DOI $10.1021 / \operatorname{ar} 9000923$

51. U. Heinemeyer, A. Hinderhofer, M.I. Alonso, J.O. Ossó, M. Garriga, M. Kytka, A. Gerlach, F. Schreiber, phys. stat. sol. a 205, 927 (2008). DOI 10.1002/pssa. 200777765

52. U. Heinemeyer, R. Scholz, L. Gisslén, M.I. Alonso, J.O. Ossó, M. Garriga, A. Hinderhofer, M. Kytka, S. Kowarik, A. Gerlach, F. Schreiber, Phys. Rev. B 78, 085210 (2008). DOI 10.1103/PhysRevB.78.085210

53. M. Birkholz, Thin Film Analysis by X-Ray Scattering (Wiley-VCH, Weinheim, 2006)

54. M. Tolan, X-ray scattering from soft-matter thin films: materials science and basic research. Springer tracts in modern physics (Springer, Berlin, 1999)

55. A. Hinderhofer, A. Gerlach, S. Kowarik, F. Zontone, J. Krug, F. Schreiber, Euro Phys. Lett. 91, 56002 (2010). DOI 10.1209/0295-5075/91/56002

56. S.R. Forrest, M.L. Kaplan, P.H. Schmidt, J. Appl. Phys. 56, 543 (1984). DOI $10.1063 / 1.333944$

57. A.C. Dürr, F. Schreiber, M. Kelsch, H.D. Carstanjen, H. Dosch, Adv. Mater. 14, 961 (2002). DOI 10.1002/1521-4095(20020705)14:13/14〈961::AID-ADMA961〉3. $0 . \mathrm{CO} ; 2-\mathrm{X}$

58. A. Hinderhofer, A. Gerlach, K. Broch, T. Hosokai, K. Yonezawa, K. Kato, S. Kera, N. Ueno, F. Schreiber, J. Phys. Chem. C 117, 1053 (2013). DOI 10.1021/jp3106056

59. A. Aufderheide, K. Broch, J. Novák, A. Hinderhofer, R. Nervo, A. Gerlach, R. Banerjee, F. Schreiber, Phys. Rev. Lett. 109, 156102 (2012). DOI 10.1103/ PhysRevLett.109.156102 
60. A. Kitaigorodsky, Mixed Crystals. Springer Series in Solid-State Sciences (Springer, Berlin, 1984)

61. K. Broch, A. Aufderheide, L. Raimondo, A. Sassella, A. Gerlach, F. Schreiber, J. Phys. Chem. C 117, 13952 (2013). DOI 10.1021/jp4019487

62. R. Banerjee, J. Novák, C. Frank, C. Lorch, A. Hinderhofer, A. Gerlach, F. Schreiber, Phys. Rev. Lett. 110, 185506 (2013). DOI 10.1103/PhysRevLett. 110.185506

63. K. Broch, A. Gerlach, C. Lorch, J. Dieterle, J. Novák, A. Hinderhofer, F. Schreiber, J. Chem. Phys. 139, 174709 (2013). DOI 10.1063/1.4827868

64. K. Broch, C. Bürker, J. Dieterle, S. Krause, A. Gerlach, F. Schreiber, phys. stat. sol. RRL 7, 1084 (2013). DOI 10.1002/pssr.201308085

65. A.K. Topczak, T. Roller, B. Engels, W. Brütting, J. Pflaum, Phys. Rev. B 89, 201203(R) (2014). DOI 10.1103/PhysRevB.89.201203

66. R.R. Lunt, J.B. Benziger, S.R. Forrest, Adv. Mater. 22, 1233 (2010). DOI 10.1002/adma.200902827

67. P. Peumans, A. Yakimov, S.R. Forrest, J. Appl. Phys. 93, 3693 (2003). DOI $10.1063 / 1.1534621$

68. R.R. Lunt, N.C. Giebrink, A.A. Belak, J.B. Benziger, S.R. Forrest, J. Appl. Phys. 105, 1233 (2009). DOI 10.1063/1.3079797

69. M. Brendel, S. Krause, A. Steindamm, A.K. Topczak, S. Sundarraj, P. Erk, S. Höhla, N. Fruehauf, N. Koch, J. Pflaum, Adv. Mater. 25, 1565 (2015). DOI 10.1002 /adfm. 201404434

70. A.C. Dürr, F. Schreiber, K.A. Ritley, V. Kruppa, J. Krug, H. Dosch, B. Struth, Phys. Rev. Lett. 90, 016104 (2003). DOI 10.1103/PhysRevLett.90.016104

71. A.K. Ghosh, T. Feng, J. Appl. Phys. 49, 5982 (1978). DOI 10.1063/1.324566

72. V. Settels, W.L. Liu, J. Pflaum, R.F. Fink, B. Engels, J. Comp. Chem. 33, 1544 (2012). DOI 10.1002/jcc. 22986

73. V. Settels, A. Schubert, M. Tafipolski, W.L. Liu, V. Stehr, A.K. Topczak, J. Pflaum, C. Deibel, R.F. Fink, V. Engel, B. Engels, J. Am. Chem. Soc. 136, 9327 (2014). DOI 10.1021/ja413115h

74. B. Gieseking, T. Schmeiler, B. Müller, C. Deibel, B. Engels, V. Dyakonov, J. Pflaum, Phys. Rev. B 90, 205305 (2014). DOI 10.1103/PhysRevB.90.205305

75. C. Schuenemann, A. Petrich, R. Schulze, D. Wynands, J. Meiss, M.P. Hein, J. Jankowski, C. Elschner, J. Alex, M. Hummert, K.J. Eichhorn, K. Leo, M. Riede, Org. Electron. 14, 1704 (2013). DOI 10.1016/j.orgel.2013.04.006

76. N.H. Hansen, C. Wunderlich, A.K. Topczak, E. Rohwer, H. Schwoerer, J. Pflaum, Phys. Rev. B 87, 241202(R) (2013). DOI 10.1103/PhysRevB.87. 241202

77. Due to the notation of the relative quenching per cent, the non-radiative recombination rate cited in ref. [76] has to be divided by a factor 100 .

78. U. Hörmann, J. Wagner, M. Gruber, A. Opitz, W. Brütting, phys. stat. sol. RRL 5, 241 (2011). DOI 10.1002/pssr.201105238

79. U. Hörmann, J. Kraus, M. Gruber, C. Schuhmair, T. Linderl, S. Grob, S. Kapfinger, K. Klein, M. Stutzman, H. Krenner, W. Brütting, Phys. Rev. B 88, 235307 (2013). DOI 10.1103/PhysRevB.88.235307

80. U. Hörmann, C. Lorch, A. Hinderhofer, A. Gerlach, M. Gruber, J. Kraus, B. Sykora, S. Grob, T. Linderl, A. Wilke, A. Opitz, R. Hansson, A.S. Anselmo, Y. Ozawa, Y. Nakayama, H. Ishii, N. Koch, E. Moons, F. Schreiber, W. Brütting, J. Phys. Chem. C 118, 26462 (2014). DOI 10.1021/jp506180k 
81. M. Horlet, M. Kraus, W. Brütting, A. Opitz, Appl. Phys. Lett. 98, 233304 (2011). DOI 10.1063/1.3598423

82. M. Gruber, J. Wagner, K. Klein, U. Hörmann, A. Opitz, M. Stutzmann, W. Brütting, Adv. Energy Mater. 2, 1100 (2012). DOI 10.1002/aenm.201200077

83. U. Rau, Phys. Rev. B 76, 085303 (2007). DOI 10.1103/PhysRevB.76.085303

84. J. Widmer, M. Tietze, K. Leo, M. Riede, Adv. Funct. Mater. 23, 5814 (2013). DOI 10.1002/adfm.201301048

85. K. Vandewal, K. Tvingstedt, A. Gadisa, O. Inganäs, J.V. Manca, Nat. Mater. 8, 904 (2009). DOI 10.1038/nmat2548

86. K. Tvingstedt, O. Malinkiewicz, A. Baumann, C. Deibel, H.J. Snaith, V. Dyakonov, H.J. Bolink, Sci. Rep. 4, 6071 (2014). DOI 10.1038/srep06071

87. R.R. King, D. Bhusari, A. Boca, D. Larrabee, X.Q. Liu, W. Hong, C.M. Fetzer, D.C. Law, N.H. Karam, Prog. Photovolt: Res. Appl. 19, 797 (2011). DOI 10.1002/pip.1044 


\section{Index}

$\alpha$-sexithiophene (6T), 4

(tris-(8-hydroxyquinoline)aluminum

(Alq3), 17

bandgap-voltage offset, 26

basic processes, 2

bottom-to-top asymmetry, 15

Buckminster fullerene

C60, 4, 13

C70, 4

charge separation, 2, 5

charge transfer energy, 4, 26

charge transfer state, 1,2

conical intersection, 17

copper phthalocyanine

$(\mathrm{CuPc}), 4$

perfluorinated (F16CuPc), 4

DFT calculation, 17

diindenoperylene (DIP), 4, 16, 19, 20

diindenoperylene derivative

tetraphenyldibenzoperiflanthene (DBP), 4, 19

tetraphenyldiindenoperylene (Ph4DIP), 19

tetrapropyltetraphenyldiindenoperylene (P4-Ph4-DIP), 19

electronic structure, 7,11

energy loss, 24-26

exciton blocking layer, 11

exciton diffusion, 15 exciton diffusion length, 2

excitonic solar cells, 1

external quantum efficiency, 3

film growth, 12

film roughness, 13

free energy of a mixture, 14

grazing incidence X-ray diffraction

(GIXD), 11

heterojunction, 1

bulk (BHJ), 5

interdigitated, 5

planar (PHJ), 5, 7

planar-mixed (PM-HJ), 6

hole injection barrier (HIB), 8

hole injection layer (HIL), 8

interface, 12

internal quantum efficiency, 2

molecular acceptors, 4

molecular donors, 4

molecular orientation, 12

non-radiative exciton-hole process, 20

open circuit voltage, 9,26

optical absorption, 2, 4

ordered complex, 15

pentacene

(PEN), 4 
32

Index

perfluorinated (PFP), 4

perylenetetracarboxylic dianhydride ( $\alpha$-PTCDA), 18

phase separation, 14,15

photoelectron spectroscopy

inverse (IPES), 7, 8

ultraviolet (UPS), 7, 8

photoluminescence quenching, 16

photovoltaic gap, 4, 9, 11, 26

potential energy landscape (PEL), 17

recombination

non-radiative, 23

radiative, 23 rate, 20

relative quenching, 17,19

Shockley-Queisser model, 1, 22

smoothing, 13

solar cell architecture, 5

solid solution, 14

structural order, 13

thin film transistor (TFT), 19

transport gap, 9

X-ray reflectivity (XRR), 11

X-ray scattering, 11 
